\title{
Asymmetric Synthesis of the Aromatic Fragment of Sespendole
}

Yoshiki Ono, Atsuo Nakazaki, Kaori Ueki, Keiko Higuchi, Uraiwan Sriphana, Masaatsu Adachi, and Toshio Nishikawa*

Graduate School of Bioagricultural Sciences, Nagoya University

Furo-cho, Chikusa, Nagoya 464-8601, Japan

E-mail: nisikawa@agr.nagoya-u.ac.jp

Contents:

1. Attempts for asymmetric synthesis of intermediates

in racemic synthesis of aromatic fragment 2 $\mathrm{S} 2-\mathrm{S} 3$

2. Comparison of ${ }^{13} \mathrm{C}-\mathrm{NMR}$ spectra

of a mixture of syn- and anti-12 and syn-12 of the literature S4

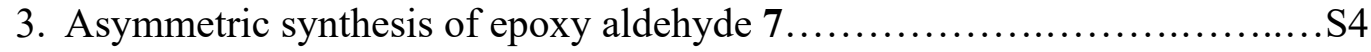

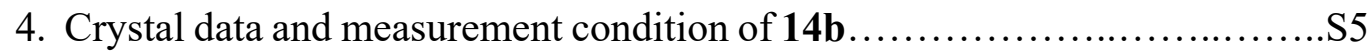

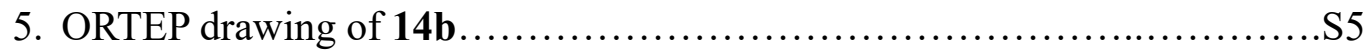

6. Spectroscopic data of the intermediates...........................S6-S20

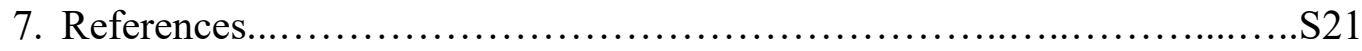


Attempts for asymmetric synthesis of intermediates in racemic synthesis of aromatic

\section{fragment 2 (Schemes S1 to S6)}

(1) An improved synthetic route of racemic aromatic fragment 2 (Scheme S1)<smiles>O=Cc1ccc([N+](=O)[O-])c(O)c1</smiles>

4

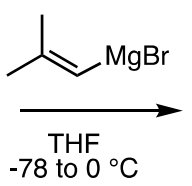

-78 to $0{ }^{\circ} \mathrm{C}$<smiles>C#CC(C)(C)Oc1cc(C(O)C2OC2(C)C)ccc1[N+](=O)[O-]</smiles>

S-3<smiles>CC(C)=CC(O)c1ccc([N+](=O)[O-])c(O)c1</smiles>

S-1

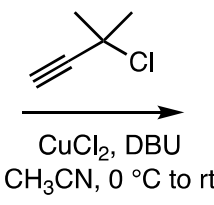<smiles>C#CC(C)(C)Oc1cc(C(O)C=C(C)C)ccc1[N+](=O)[O-]</smiles>

S-2

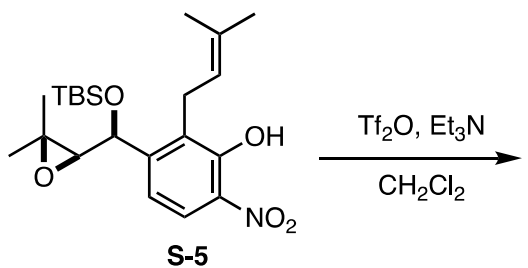

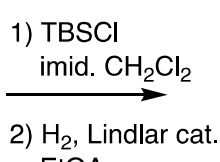
EtOAc<smiles>C=CC(C)(C)Oc1cc(C([OH2+])C2OC2(C)C)ccc1[N+](=O)[O-]</smiles>

S-4<smiles>COC(c1ccc([N+](=O)[O-])c(O)c1CC=C(C)C)C1OC1(C)C</smiles>

aromatic fragment 2

\section{Scheme S1}

(2) Unsuccessful attempts for preparing chiral intermediates of the racemic synthesis of 2 All the optical purities indicated below were determined by chiral HPLC analysis.

(2-a) Kinetic resolution of $( \pm)$ S-2 by Sharpless asymmetric epoxidation ${ }^{1}$ (Scheme S2)<smiles>C#CC(C)(C)Oc1cc([C@@H](O)C=C(C)C)ccc1[N+](=O)[O-]</smiles>

S-2

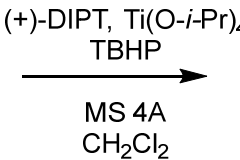

(48\% conversion)<smiles>C#CC(C)(C)Oc1cc(C(O)C=C(C)C)ccc1[N+](=O)[O-]</smiles>

S-2 $56 \%$ ee

\section{Scheme S2}

Kinetic resolution of $( \pm)$ S-1 by lipase-catalyzed acetylation (Scheme S3)<smiles>CC(C)=C[C@@H](O)c1ccc([N+](=O)[O-])c(O)c1</smiles>

S-1

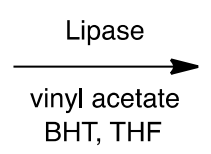

Lipase TL (33 h, 57\% conversion ) Lipase QLM (24h, 30\% conversion)<smiles>CC(C)=CC(O)c1ccc([N+](=O)[O-])c(O)c1</smiles>

$30 \%$ ee $26 \%$ ee<smiles>[CH2+]C(C)=CC(OC(C)=O)c1ccc([N+](=O)[O-])c(O)c1</smiles>

$19 \%$ ee $60 \%$ ee

\section{Scheme S3}

No acetylation was observed in the same acetylation by other lipases such as Amano PS, SL, AK, PL. 
(2-b) CBS reductions ${ }^{2}$ of enone $\mathbf{S - 6}$ prepared by oxidation of $( \pm)$ S-2 (Scheme S4)<smiles>C#CC(C)(C)Oc1cc(C(=O)C=C(C)C)ccc1[N+](=O)[O-]</smiles>

S-6

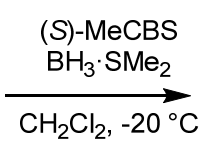

-

Scheme S4

(2-c) Asymmetric epoxidation of enone S-6 under Ender's conditions ${ }^{3}$ (Scheme S5)<smiles>C#CC(C)(C)Oc1cc(C(=O)C=C(C)C)ccc1[N+](=O)[O-]</smiles>

S-6

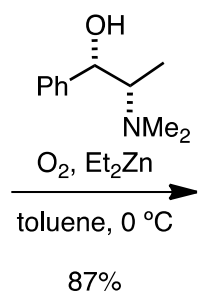

$87 \%$

Scheme S5

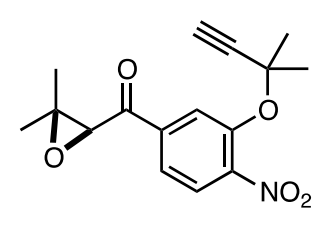

S-7 $28 \%$ ee<smiles>C#CC(C)(C)Oc1cc(C(O)C=C(C)C)ccc1[N+](=O)[O-]</smiles>

S-3 $10 \%(0.5 \%$ ee $)$ 
Comparison of ${ }^{13} \mathrm{C}-\mathrm{NMR}$ spectra of a mixture of syn- and anti-12 and syn-12 of the literature (Table S1). ${ }^{4}$

\section{Table S1}

\begin{tabular}{ccc} 
major $(\Delta \delta)$ & lit. $(s y n)^{4}$ & minor $(\Delta \delta)$ \\
\hline $19.7(+0.1)$ & 19.6 & $18.9(-0.7)$ \\
$24.9(+0.1)$ & 24.8 & $24.8( \pm 0.0)$ \\
$60.1(+0.1)$ & 60.0 & $59.7(-0.3)$ \\
$68.0( \pm 0.0)$ & 68.0 & $66.5(-1.5)$ \\
$72.6( \pm 0.0)$ & 72.6 & $72.4(-0.2)$ \\
$126.0(+0.1)$ & 125.9 & $126.0(+0.1)$ \\
$128.1(+0.1)$ & 128.0 & $128.1(+0.1)$ \\
$128.7(+0.1)$ & 128.6 & $128.6( \pm 0.0)$ \\
$140.1( \pm 0.0)$ & 140.1 & $141.5(+1.4)$ \\
\hline & & $(p p m)$
\end{tabular}

Asymmetric synthesis of epoxy aldehyde 7 (Scheme S7)

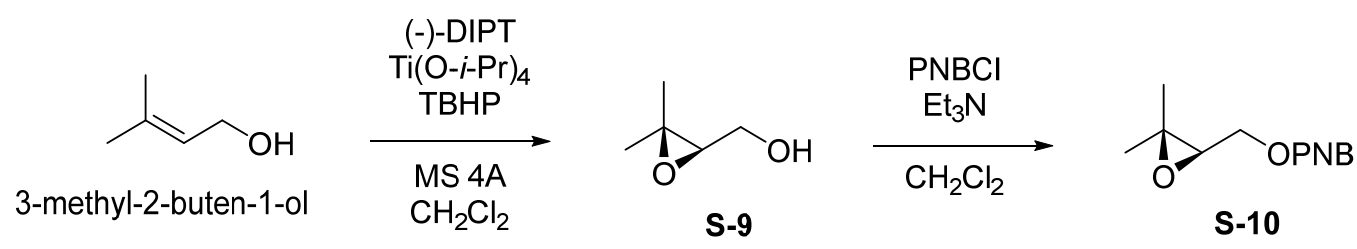

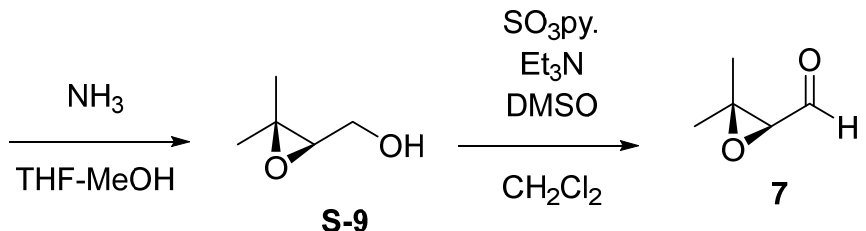

Scheme S7 


\section{Crystal data and measurement condition of $14 \mathrm{~b}$}

Table S2. Crystal data and measurement condition of $\mathbf{1 4 b}$

\begin{tabular}{|c|c|c|c|}
\hline (a) Crystal Data & \multicolumn{3}{|c|}{ (b) Intensity Measurements } \\
\hline Emprical Formula & $\mathrm{C}_{18} \mathrm{H}_{15} \mathrm{BrN}_{2} \mathrm{O}_{6}$ & Diffractometer & Rigaku R-AXIS RAPID \\
\hline Formula Weight & 435.23 & Radiation; & $\mathrm{CuK \alpha}$ \\
\hline Crystal System & triclinic & $\lambda(\AA)$ & 1.54187 \\
\hline Space Group & $\mathrm{P}-1(\# 2)$ & & \\
\hline $\mathrm{a}(\AA)$ & $8.3957(5)$ & & \\
\hline $\mathrm{b}(\AA)$ & $10.1273(6)$ & \multicolumn{2}{|l|}{ (c) refinement } \\
\hline c $(\AA)$ & $10.8640(6)$ & Data & 3256 \\
\hline $\mathrm{V}\left(\AA^{3}\right)$ & $913.18(9)$ & / Parameters & 1245 \\
\hline$\alpha(\operatorname{deg})$ & 94.618 & Goodnecs-of fit & 1099 \\
\hline$\beta(\operatorname{deg})$ & 95.332 & Foouness-ot ith & $\mathrm{D}-0.0567$ \\
\hline$\gamma(\operatorname{deg})$ & 94.579 & \multirow{2}{*}{\multicolumn{2}{|c|}{$[\mathrm{I}>2 \sigma(\mathrm{I})] \mathrm{wR}_{2}=0.1637$}} \\
\hline$Z$ value & 2 & & \\
\hline Temperature (K) & 173 & & \\
\hline
\end{tabular}

\section{ORTEP drawing of 14b}

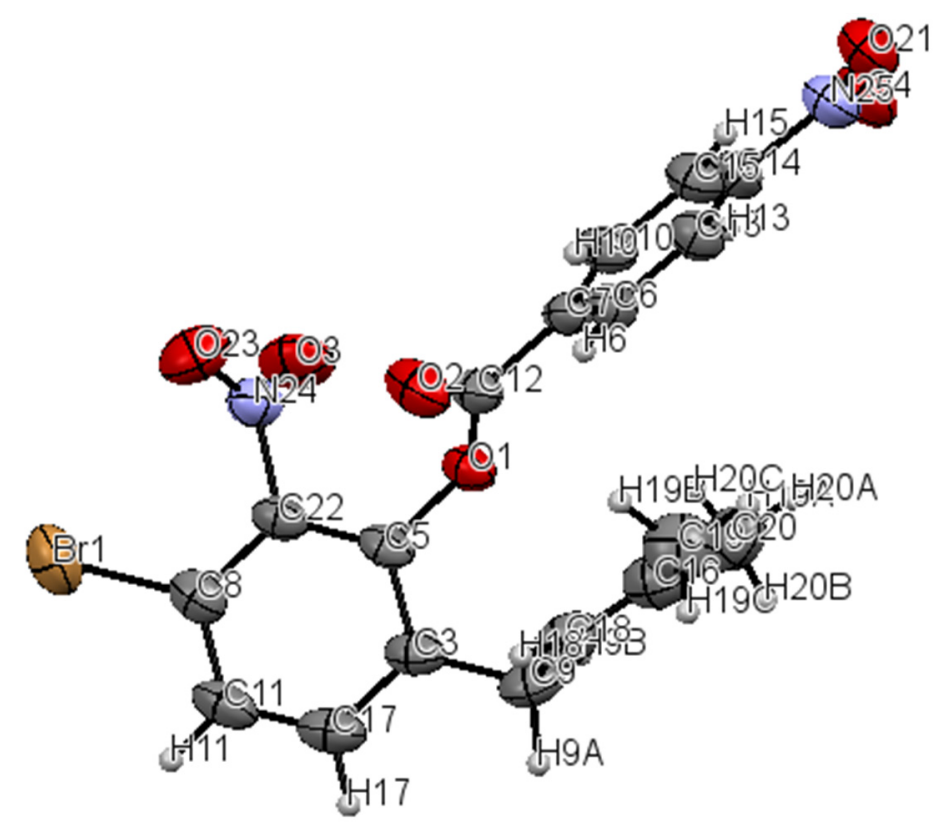

Figure S1. ORTEP diagram of 14b (CCDC- 1878380). The thermal ellipsoid probabilities are shown at $50 \%$. Compound 14b was recrystallized from $\mathrm{Et}_{2} \mathrm{O}$ at $\mathrm{rt}$ to afford a single crystal that was suitable for $\mathrm{X}$-ray analysis. 

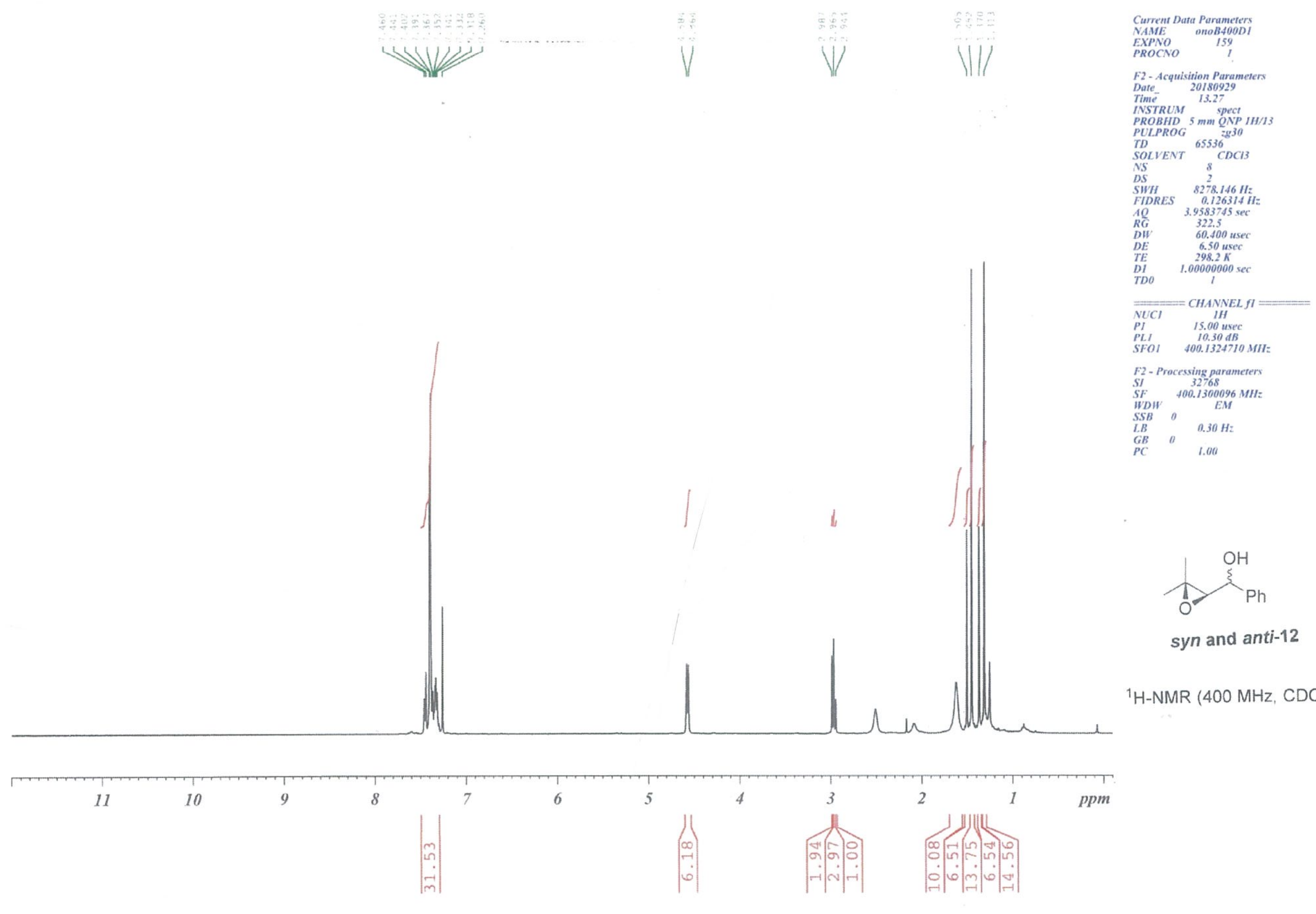

${ }^{1} \mathrm{H}-\mathrm{NMR}\left(400 \mathrm{MHz}, \mathrm{CDCl}_{3}\right)$

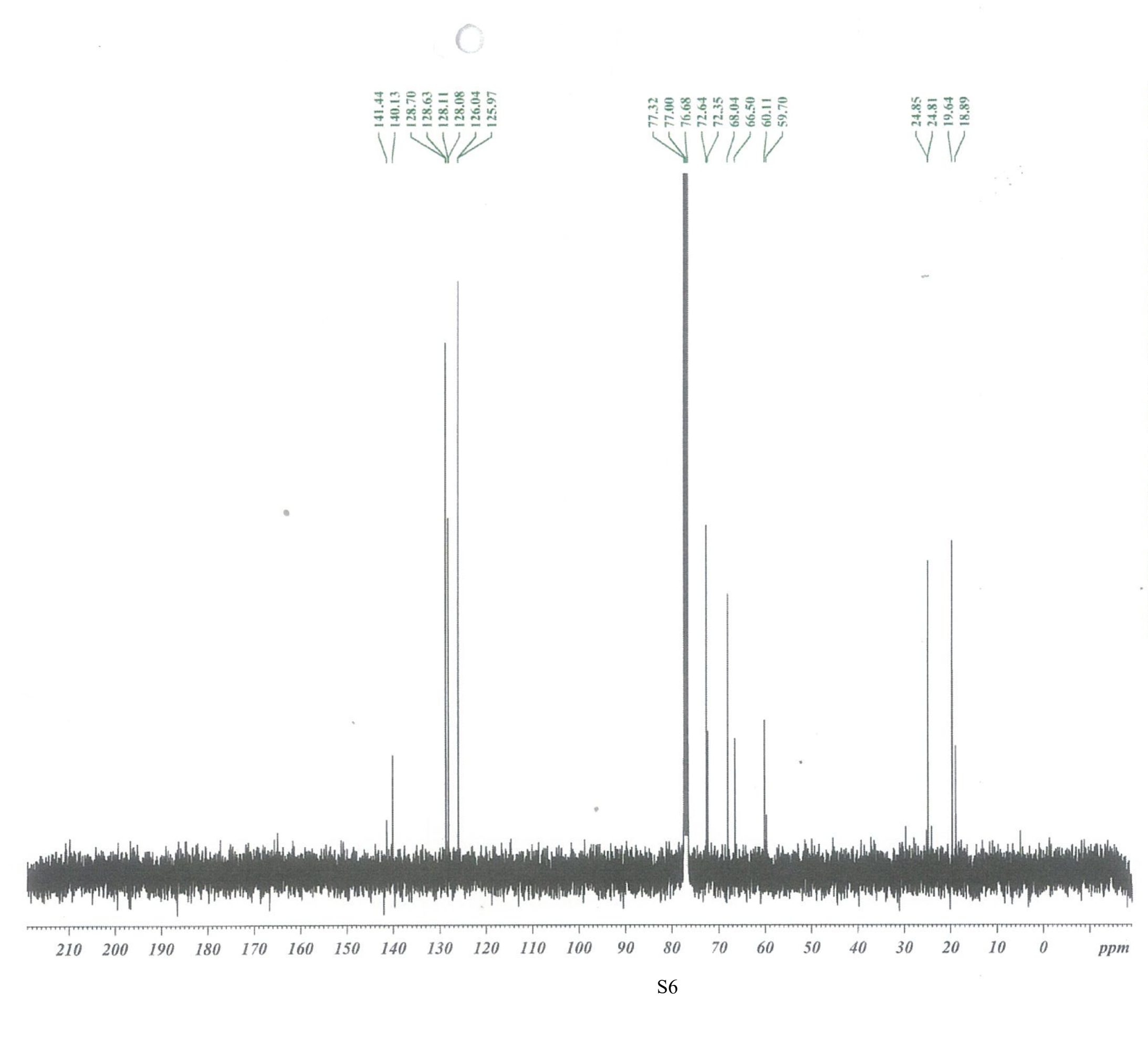

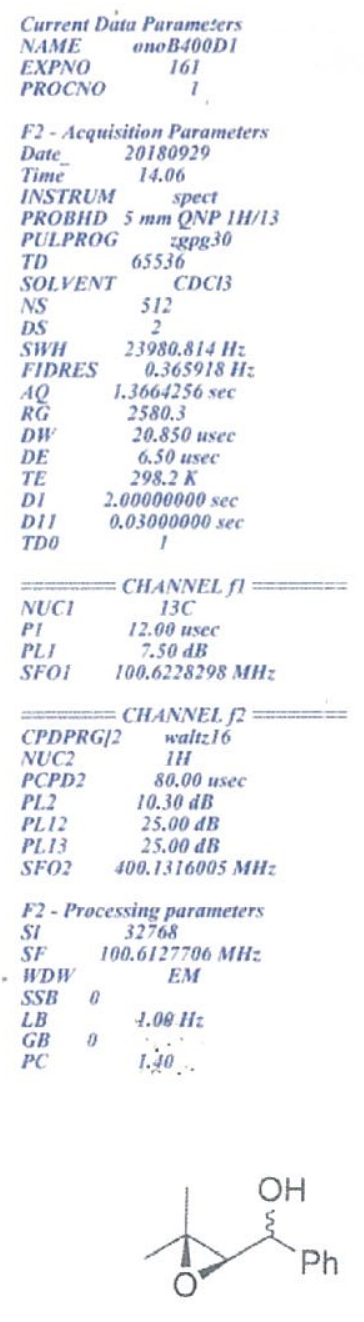

syn and anti-12

${ }^{13} \mathrm{C}-\mathrm{NMR}\left(100 \mathrm{MHz}, \mathrm{CDCl}_{3}\right.$ ) 


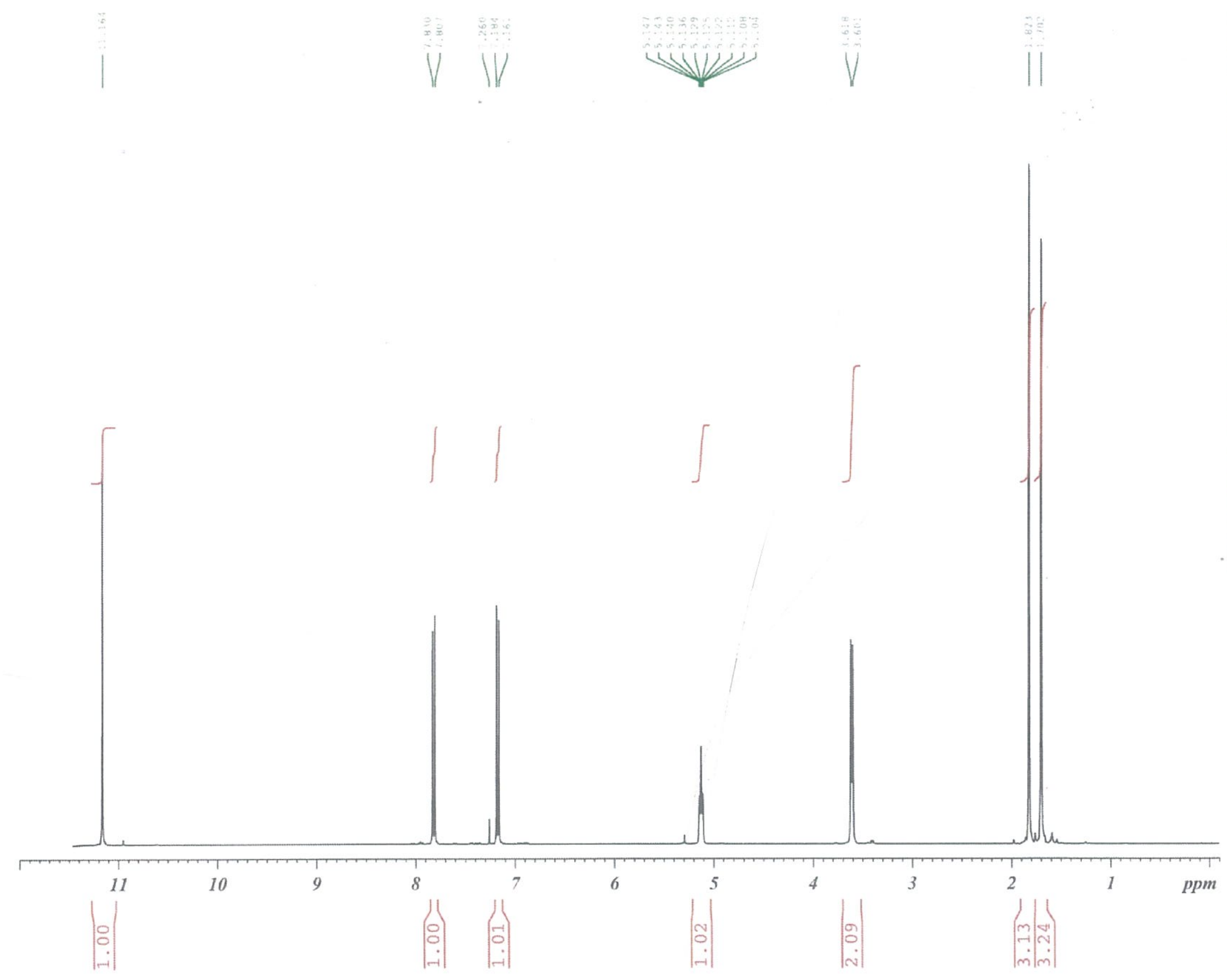

Current Data Parameters

EXPNO

F2 - Acquisition Parameter

Timé $\quad 19.33 \mathrm{~h}$

INSTRUM spect

PROBHD $5 \mathrm{~mm} Q \mathrm{QNP} / \mathrm{H} / 3$

zg30

SOLVENT ${ }^{65536} \mathrm{CDCl}$

$\begin{array}{ll}N S & 8 \\ D S & 2\end{array}$

SWH $\quad 4901.961 \mathrm{~Hz}$

HoRes $0.6846719 \mathrm{sec}$

$\begin{array}{ll}A \bar{Q} & 6.684678 \\ R & 71.8\end{array}$

DW $\quad 102.000$ usec

$D E \quad 6.50$ usec

$\begin{array}{ll}T E & 297.1 \mathrm{~K} \\ D I & 1.0000000 \mathrm{sec}\end{array}$

TDO $\quad 1.00000000 \mathrm{sec}$

$\begin{array}{ll}\text { TDO } & 1 \\ \text { SFOI } & 400.1321477 \mathrm{MHz}\end{array}$

$\begin{array}{ll}N \text { NUI } & 1 \mathrm{H} \\ \mathrm{PI} & 15.00 \mathrm{usec}\end{array}$

F2 - Processing parameters

$\begin{array}{ll}S I & 32768 \\ S F & 400.1300122 \mathrm{MHz}\end{array}$

WDW $\quad \begin{aligned} & 400.1300122 \mathrm{M} \\ & \text { EM }\end{aligned}$

$\begin{array}{lll}S S B & 0 & \\ L B & 0.30 \mathrm{~Hz}\end{array}$

$\begin{array}{lll}G B & 0 & \\ P C & & 1.00\end{array}$

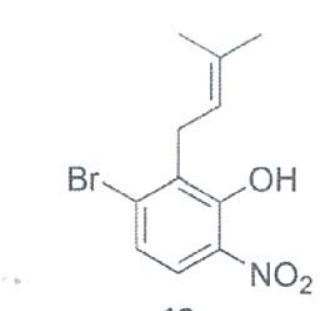

13

${ }^{1} \mathrm{H}-\mathrm{NMR}\left(400 \mathrm{MHz}, \mathrm{CDCl}_{3}\right)$

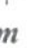

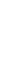

$$
\text { (n) }
$$
$\begin{array}{lc}\text { Current Data } & \text { Parameters } \\ \text { NAME } & \text { ono A400 } \\ \text { EXPNO } & 85 \\ \text { PROCNO } & 1\end{array}$

F2 - Acquisition Parameter

Date_ 20161201

Time-
INSTRUM

INSTRUM spect

PROBHD $5 \mathrm{MT}$ QNP IHII3

PULPROG
TD
$6536 \mathrm{gpg} 30$

SOLVENT ${ }^{C D C l}$

$\begin{array}{lc}\text { NS } & 256 \\ D S & 2\end{array}$

$\begin{array}{ll}D S & 2 \\ S W H & 23980.814 \mathrm{~Hz}\end{array}$

$\begin{array}{ll}\text { SWD } & 23980.814 \mathrm{~Hz} \\ \text { FIDRES } & 0.731836 \mathrm{~Hz}\end{array}$

AQ $\quad 1.3664256 \mathrm{sec}$

$\begin{array}{ll}A Q & 1.36742 .7 \\ R G & 574.850\end{array}$

$\begin{array}{ll}D W & 20.850 \text { usec } \\ D E & 6.50 \text { usec }\end{array}$

$\begin{array}{ll}D E & 6.50 \text { usec } \\ \text { TE } & 297.9 \mathrm{~K}\end{array}$

DI $1.00000000 \mathrm{sec}$

dil $0.03000000 \mathrm{sec}$

DELTA $0.89999998 \mathrm{sec}$

$\begin{array}{ll}\text { TDO } & 1 \\ \text { SFO } & 100.6228298 \mathrm{MHz}\end{array}$

SFOI $100.6228298 \mathrm{MH}$

${ }_{P I} \quad 10.00 \mathrm{C}$

$\begin{array}{lc}\text { PI } & 10.00 \mathrm{usec} \\ \text { SFO2 } & 400.1316005 \mathrm{MHz}\end{array}$

NUC2 $1 \mathrm{H}$

$\begin{array}{ll}\text { CPDPRG/2 } & \text { waltz } 16 \\ \text { PCPD2 } & 80.00 \text { usec }\end{array}$

F2 - Processing parameters
$S I$

$\begin{array}{ll}S F & 32768 \\ S F & 100.6127755 \mathrm{MH}\end{array}$

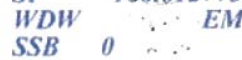

$\begin{array}{lll}L B & & 1.00 \mathrm{H} \\ \mathrm{GB} & 0 & \\ P C & & 1.40\end{array}$

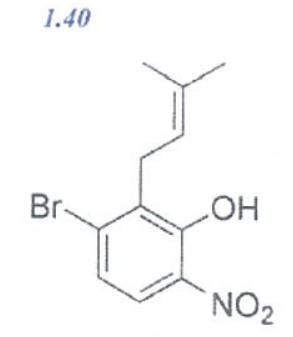

13

${ }^{13} \mathrm{C}-\mathrm{NMR}\left(100 \mathrm{MHz}, \mathrm{CDCl}_{3}\right)$ 

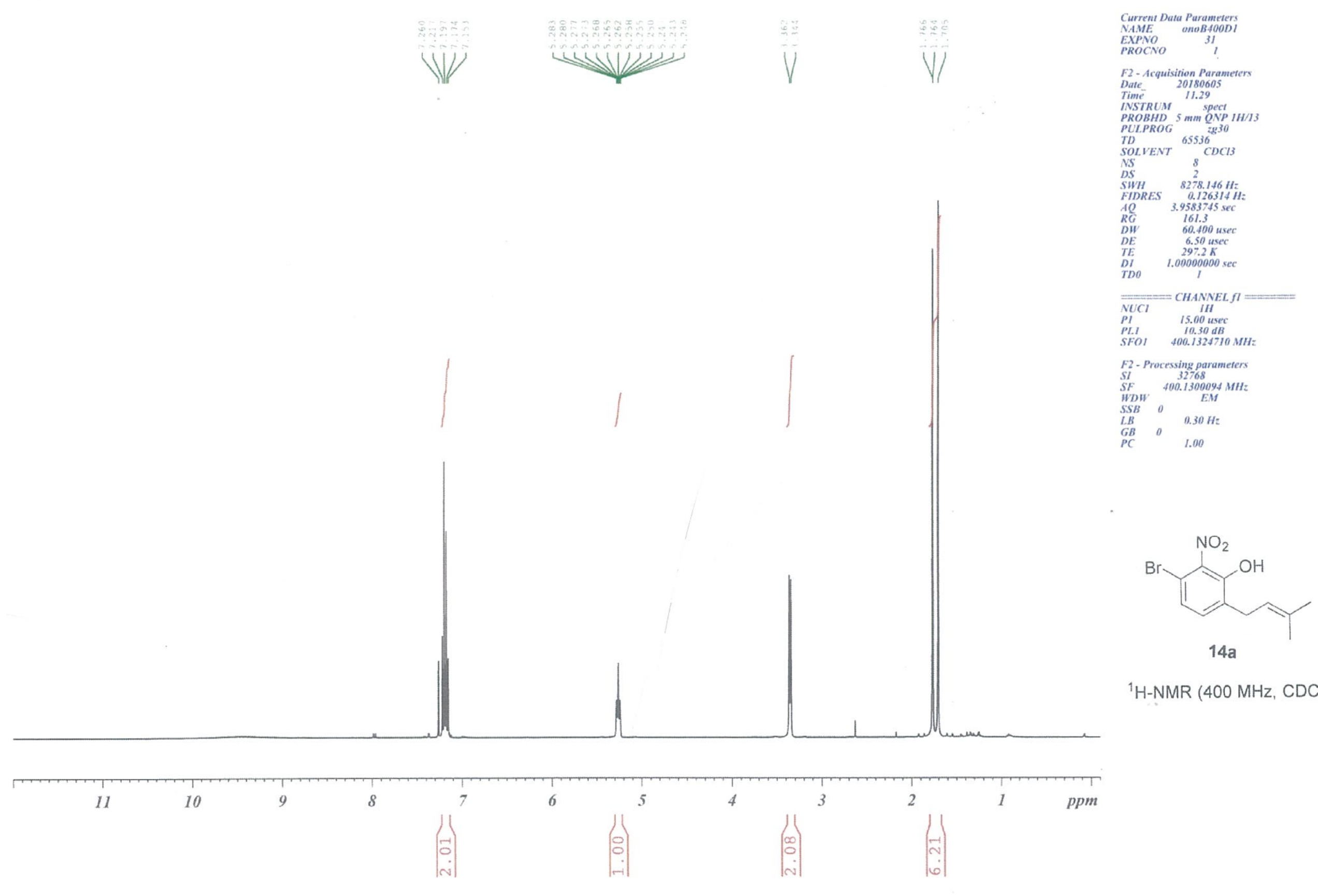

${ }^{1} \mathrm{H}-\mathrm{NMR}\left(400 \mathrm{MHz}^{-\mathrm{CDCl}_{3}}\right)$
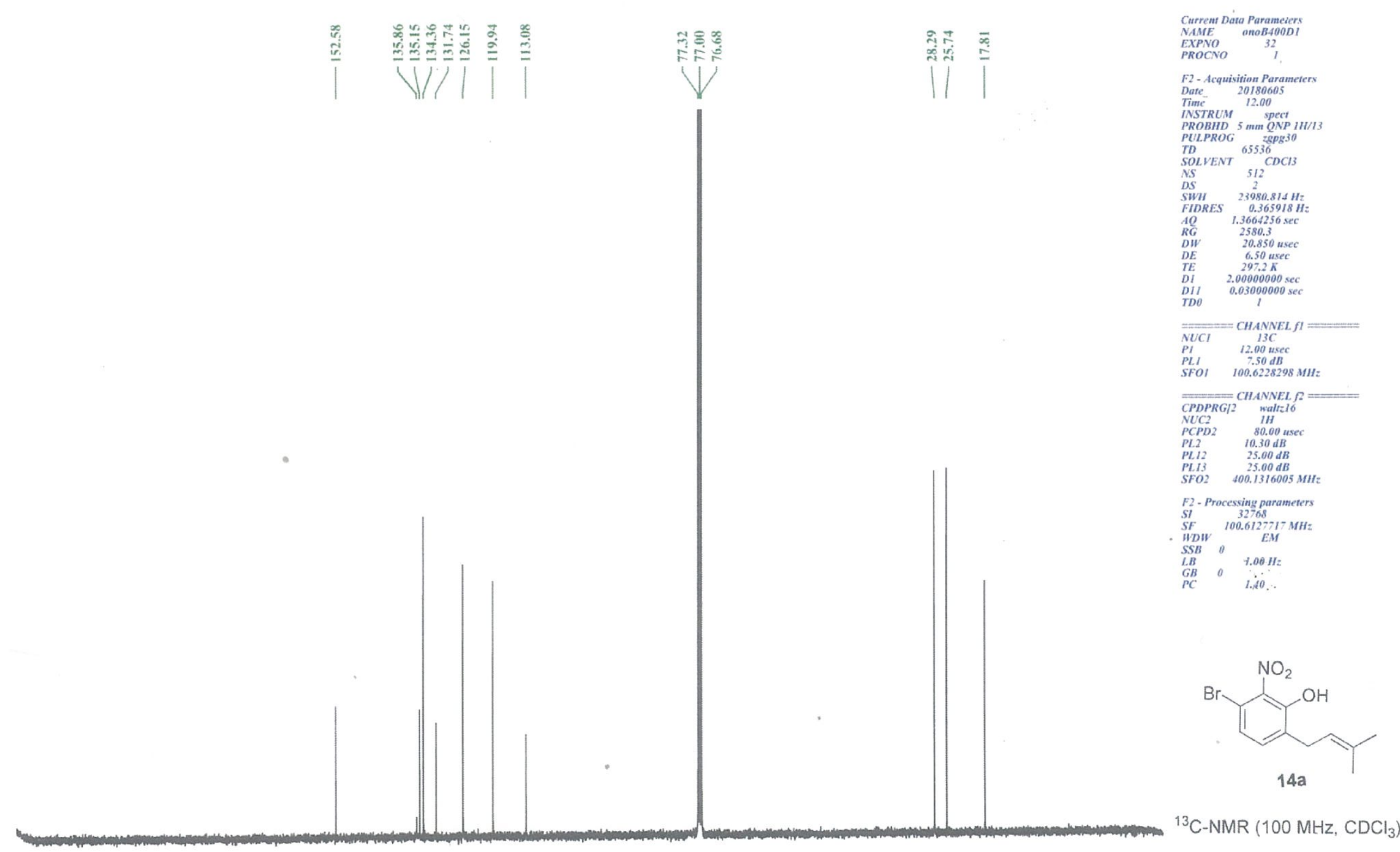

${ }^{13} \mathrm{C}-\mathrm{NMR}\left(100 \mathrm{MHz}, \mathrm{CDCl}_{3}\right)$

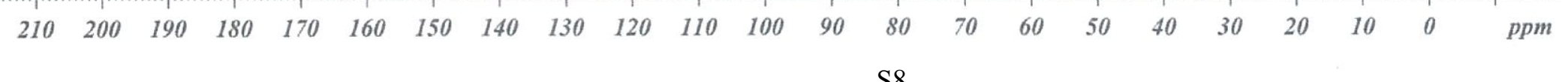



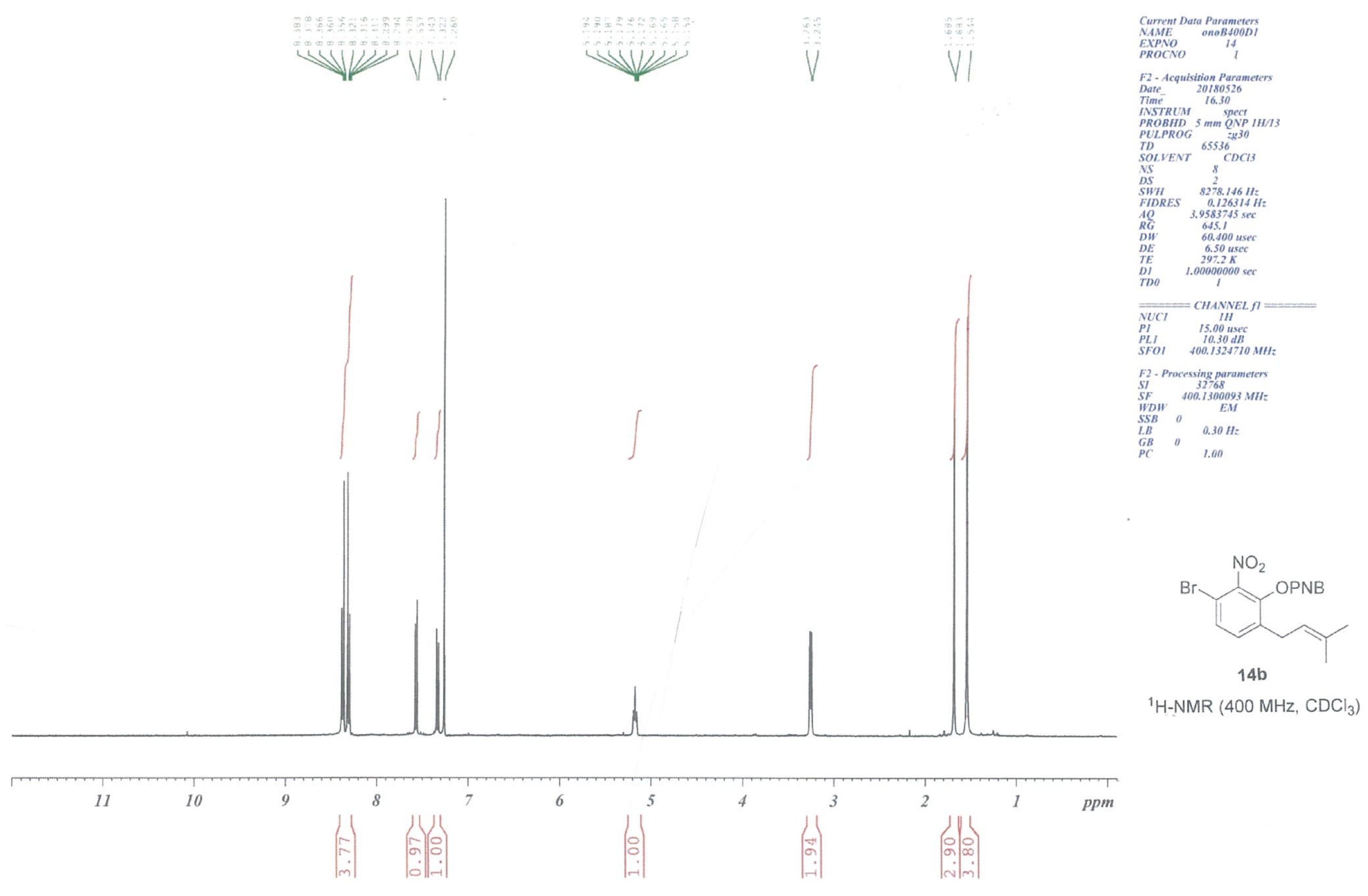

$180601 \mathrm{BP}$

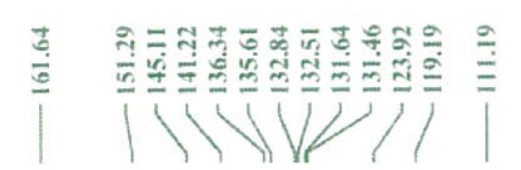

要要

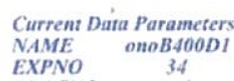

ROCNO $\quad$.

F2- Acquisition Paramet
Date-
Tite 20180607
Tims
19.13

Time
INSTRUM spect
PROBHD $5 \mathrm{~mm}$ QNP IH/13

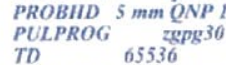

SOLVENT ${ }^{65336}$ CDCI

$\begin{array}{ll}\text { DS } & 1024 \\ \text { SWH } & 23980.814 \mathrm{H}\end{array}$

\begin{tabular}{ll} 
IDRES & 0.365918 \\
\hline$Q$ & $1.366 .256 \mathrm{sec}$
\end{tabular}

$\begin{array}{ll}R G & 2048 \\ D W & 20.850 \text { usec } \\ D E & 6.50 \text { usec } \\ D E & 297.2 \mathrm{~K}\end{array}$

$\begin{array}{ll}\text { TE } & 29.2 .2 \mathrm{~K} \\ D I & 2.00000000 \mathrm{sec} \\ D I 1 & 0.03000000 \mathrm{sec}\end{array}$

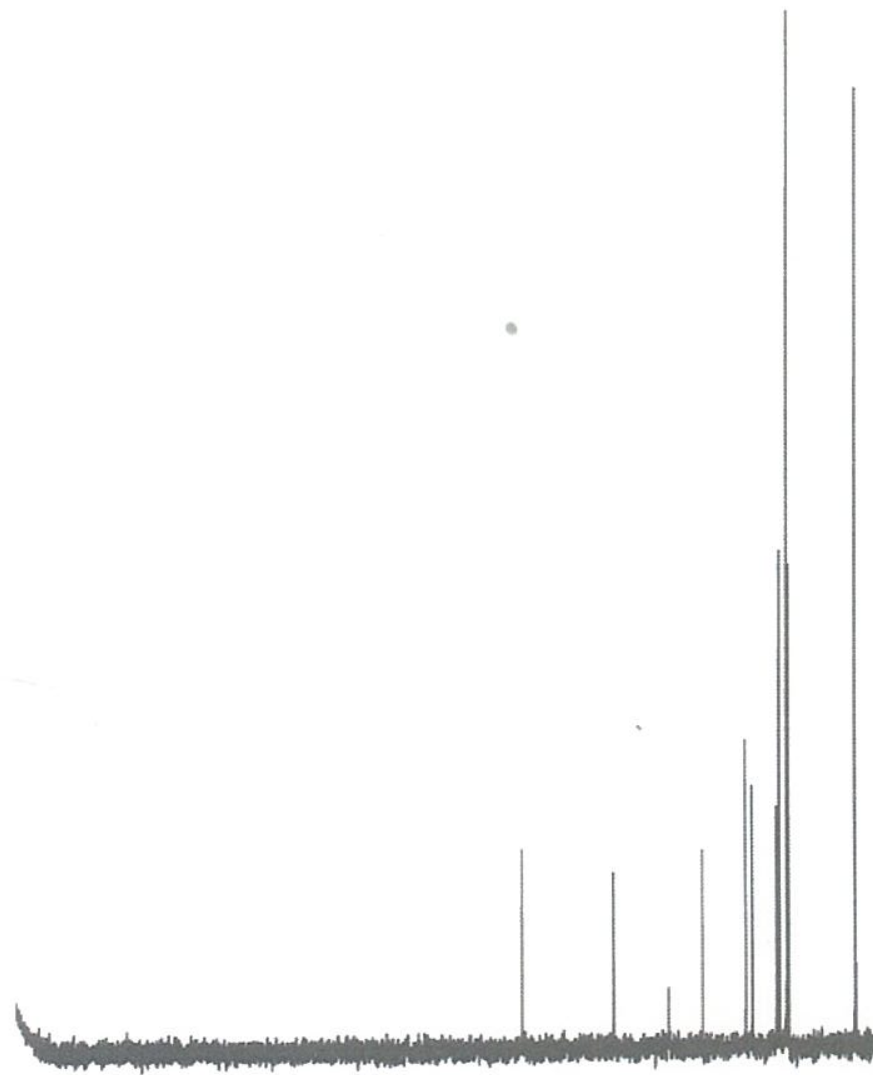

$={ }_{13 C}=$

$\begin{array}{lc}\text { PI } & 12.00 \mathrm{usec} \\ \text { PLI } & 7.50 \mathrm{~dB} \\ \text { SFOT } & 100.6228298 \mathrm{MH}\end{array}$

$\overline{\text { CPDPRG/2 }}$ CHANNEL $/ 2$

$\begin{array}{lc}\text { CPDPRG/2 } & \text { waltz/6 } \\ \text { NUC2 } & \text { ut } \\ \text { PCPD2 } & \text { so.0o usec }\end{array}$

$\begin{array}{ll}P L 2 & 10.30 \mathrm{~dB} \\ P L 12 & 25.00 \mathrm{~dB}\end{array}$

$\begin{array}{ll}\text { PLI3 } & 25.00 \mathrm{~dB} \\ \text { SFO2 } & 400.1316005 \mathrm{MHz}\end{array}$

$\mathrm{F} 2$ - Processing parameters
$S I$

WDW ${ }_{E M}$

$\begin{array}{lll}S S B & 0 \\ L B & 1.00 \mathrm{~Hz}\end{array}$

$P C \quad 1.90$.

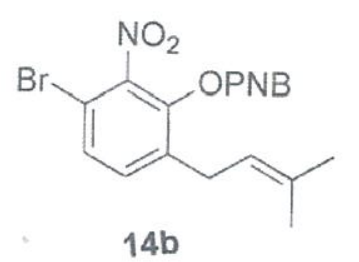

${ }^{13} \mathrm{C}-\mathrm{NMR}\left(100 \mathrm{MHz}, \mathrm{CDCl}_{3}\right.$ )

$\begin{array}{lllllllllllll}210 & 200 & 190 & 180 & 170 & 160 & 150 & 140 & 130 & 120 & 110 & 100 & 90\end{array}$ 


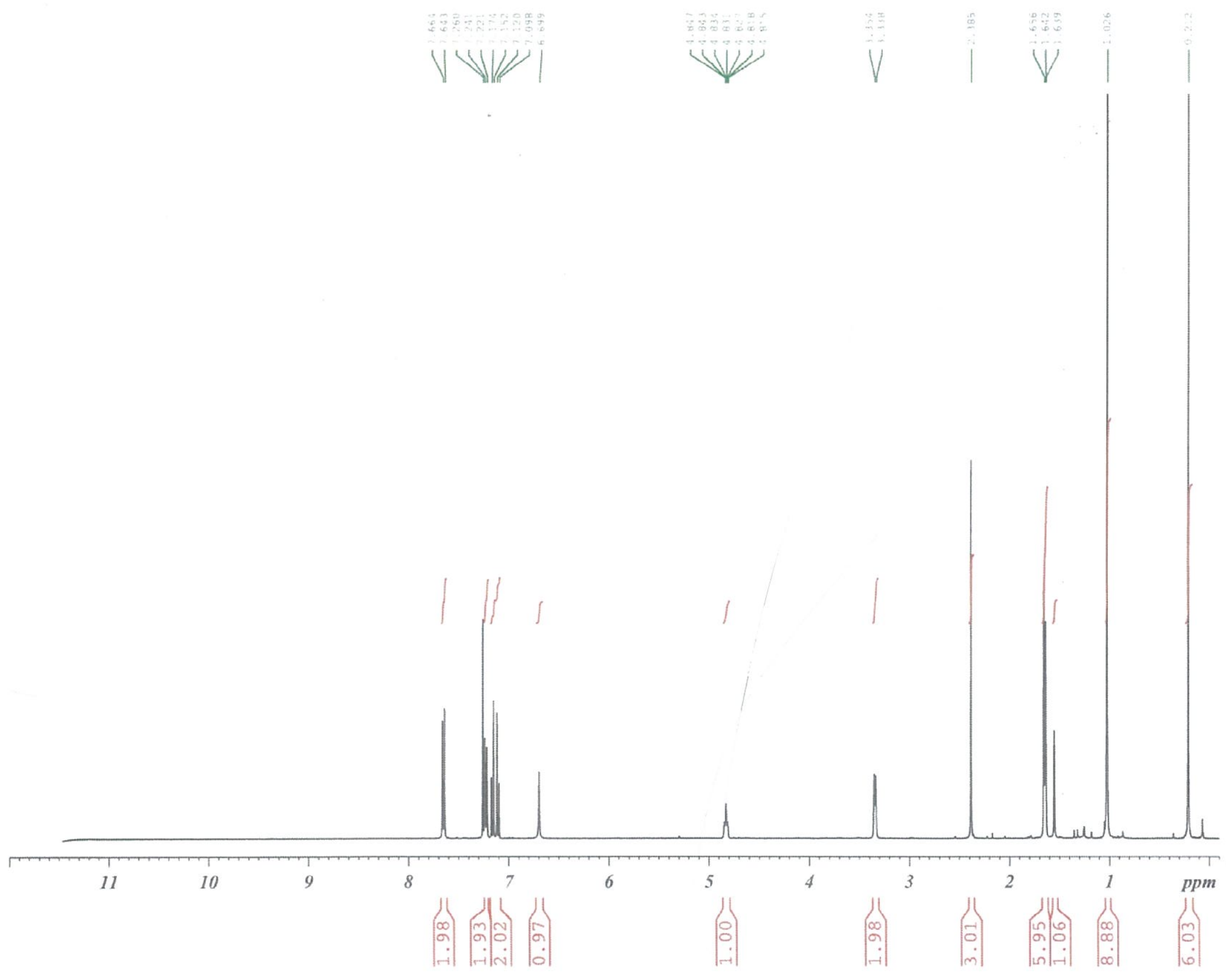

Current Data Parameters
NAME ono A400

EXPNO 96

F2 - Acquisition Parameters

Date 20161220

INSTRUM spect

PROBHD $5 \mathrm{~mm}$ QNP IH/I3

PULPROG ${ }_{\text {TD }}$ ig30
65536

SOLVENT $\quad$ CDCl

$\begin{array}{lc}\text { NS } & 8 \\ D S & 2 \\ \text { SWH } & 4901.961 \mathrm{~Hz}\end{array}$

SWH
FIDRES

$\begin{array}{lc}A Q & 6.6846719 \mathrm{sec}\end{array}$

DW $\quad 102.000$ usec

$\begin{array}{ll}D E & 6.50 \mathrm{usec} \\ T E & 297.3 \mathrm{~K}\end{array}$

$\begin{array}{lc}T E & 297.3 \mathrm{~K} \\ D I & 1.00000000 \mathrm{sec}\end{array}$

$\begin{array}{lc}\text { TDO } & 1 \\ \text { SFOI } & 400.1321477 \mathrm{MH}\end{array}$

$\begin{array}{lr}\text { NUCl } & 1 \mathrm{H} \\ \text { Pl } & 15.00 \text { usec }\end{array}$

F2 - Processing parameters

$\begin{array}{ll}S I & 32768 \\ S F & 400.1300096 \mathrm{MHz}\end{array}$

WDW $\quad E M$

$\begin{array}{lll}S B & 0.30 \mathrm{~Hz}\end{array}$

$\begin{array}{lll}L B & 0 & 0.30 \\ P C & & 1.00\end{array}$

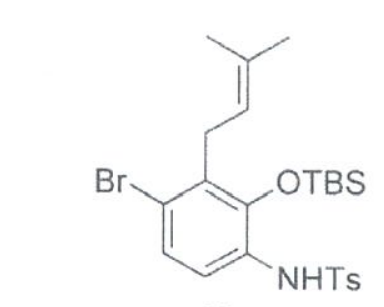

16

${ }^{1} \mathrm{H}-\mathrm{NMR}\left(400 \mathrm{MHz}, \mathrm{CDCl}_{3}\right)$

固瞙自

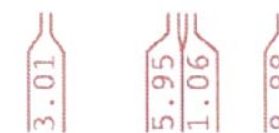

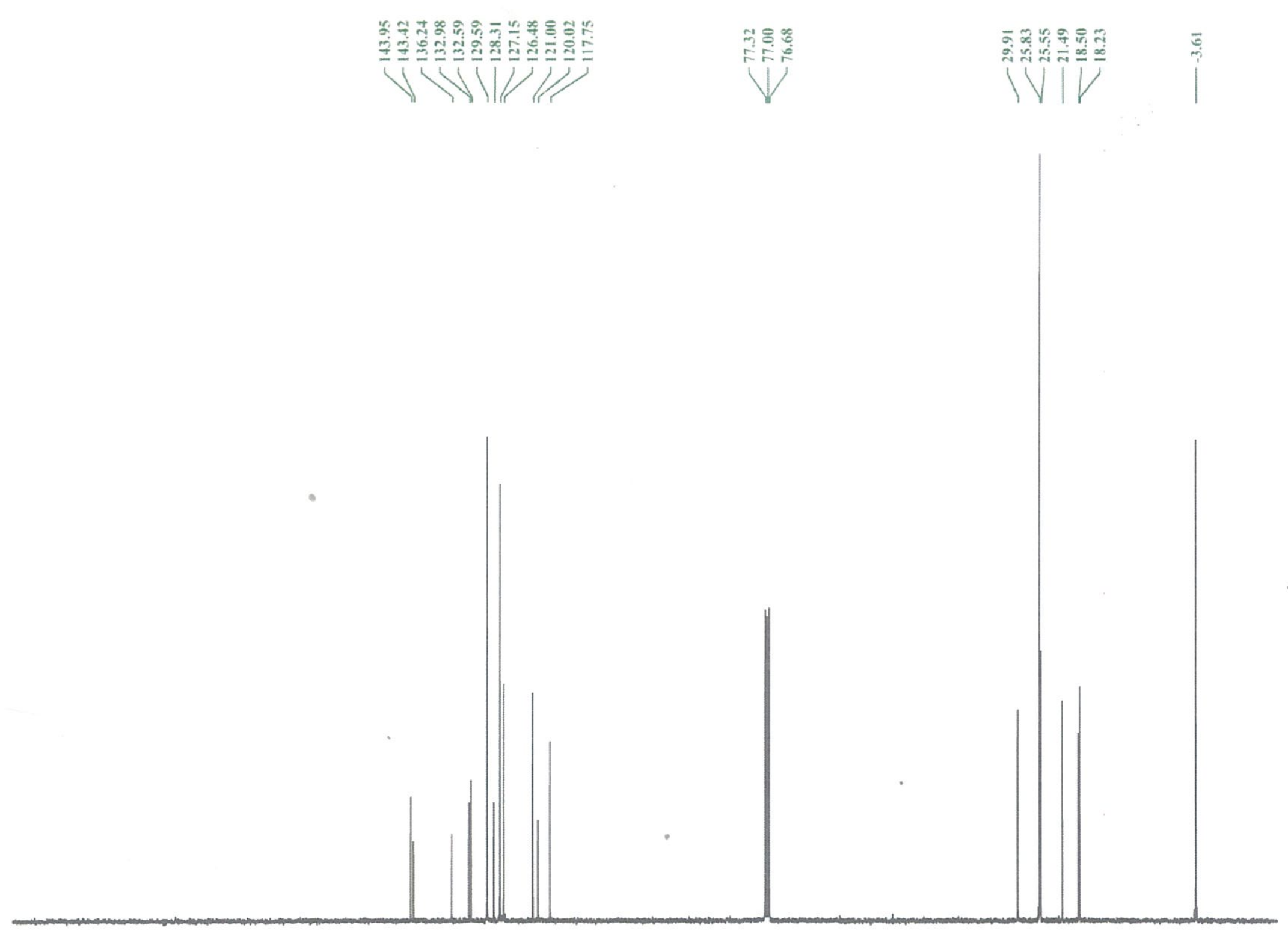

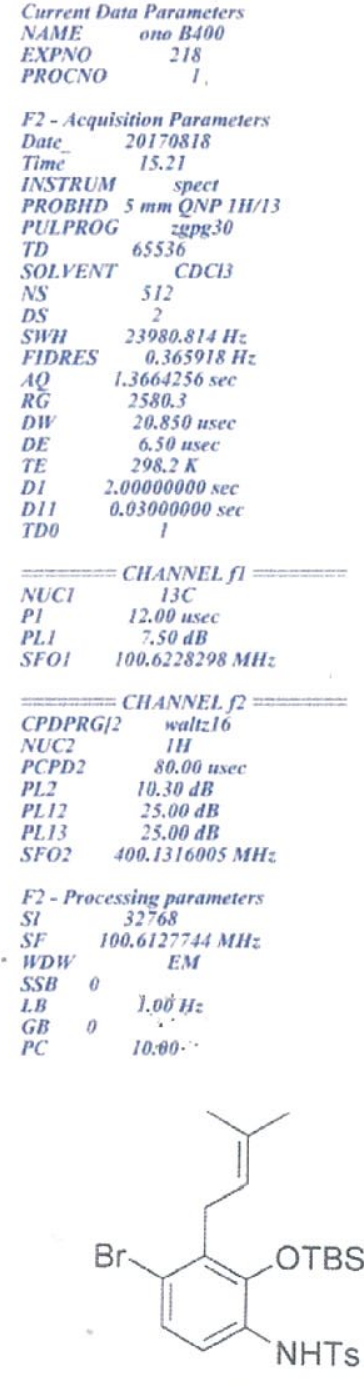

16

${ }^{13} \mathrm{C}-\mathrm{NMR}\left(100 \mathrm{MHz}, \mathrm{CDCl}_{3}\right.$ ) 


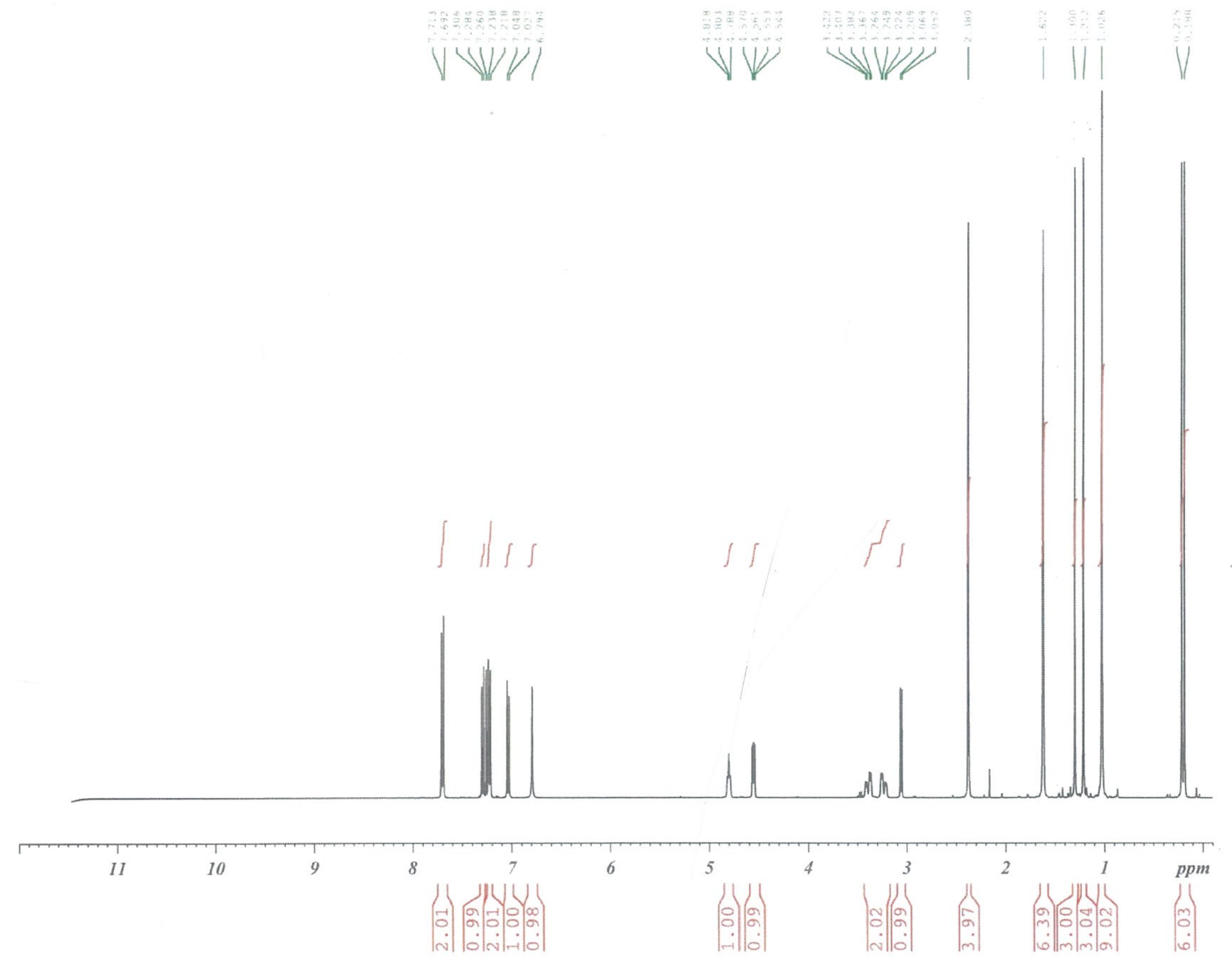

Current Data Parameters
NAME ono A400

EXPNO $\quad 163$

F2 - Acquisition Parameters

Date
Time
20170215
$18.23 \mathrm{~h}$

INSTRUM spect

TM QNP $1 \mathrm{H} / 13$

${ }_{T D}{ }_{65536}^{2830}$

SOLVENT CDC13

$\begin{array}{ll}N S & 8 \\ D S & 2\end{array}$

SWH $\quad 4901.961 \mathrm{~Hz}$

FIDRES $0.149596 \mathrm{H}$

$\begin{array}{ll}A Q & 128 \\ D G & 102.000\end{array}$

DW $\quad 102.000$ usec

TE $\quad 297.9 \mathrm{~K}$

DI $\quad 1.00000000 \mathrm{sec}$

$\begin{array}{ll}\text { TDO } & 1 \\ \text { SFOI } & 400.1321477 \mathrm{MHz}\end{array}$

$\begin{array}{cc}\mathrm{NUCl} & \mathrm{IH} \\ \mathrm{PI} & 15.00 \mathrm{usec}\end{array}$

F2 - Processing parameters

$\begin{array}{ll}S I & 32768 \\ S F & 400.1300096 \mathrm{MHz}\end{array}$

SSB 0

$\begin{array}{lll}L B & & 0.30 \mathrm{~Hz}\end{array}$

$\begin{array}{lll}G B & 0 & \\ P C & & 1.00\end{array}$

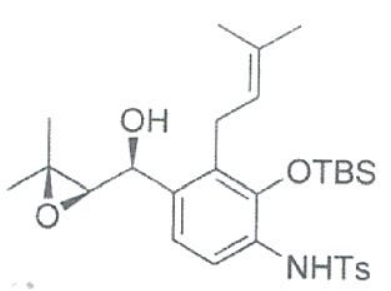

$20 a$

${ }^{1} \mathrm{H}-\mathrm{NMR}\left(400 \mathrm{MHz}, \mathrm{CDCl}_{3}\right)$

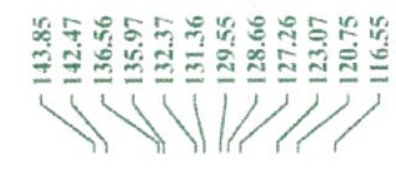

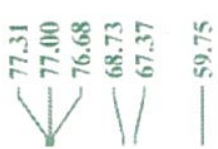

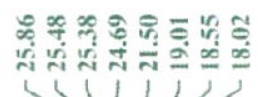

$4=$

Current Data Parameters
NAME ono A400

EXPNO $\quad 165$

F2 - Acquisition Parameters

$\begin{array}{cr}\text { Date } & 20170215 \\ \text { Time } & 19.02 \mathrm{~h}\end{array}$

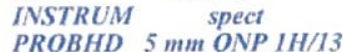

PROBPROG $5 \mathrm{~mm}$ QN ${ }^{\text {PNP } 30}$

TD 65536

SOLVENT CDCl.

$\begin{array}{lc}N S & 256 \\ D S & 2\end{array}$

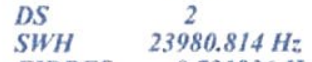

FIDRES $0.731836 \mathrm{~Hz}$

${ }_{R}^{A Q} \quad 1.3664256$

$\begin{array}{ll}D W & 20.850 \text { usec } \\ D E & 6.50 \text { usec }\end{array}$

$\begin{array}{ll}D E & 6.50 \mathrm{usec} \\ T E & 298.3 \mathrm{~K}\end{array}$

DI $\quad 1.00000000 \mathrm{sec}$

DELTA $0.89999998 \mathrm{sec}$

$\begin{array}{lc}\text { TDO } & l \\ \text { SFOI } & 100.6228298 \mathrm{MHz}\end{array}$

NUCl $\quad 13 \mathrm{C}$

$\begin{array}{lc}\text { P1. } & 10.00 \text { usec } \\ \text { SFO2 } & 400.1316005 \mathrm{MHz}\end{array}$

NUC2 $1 \mathrm{H}$

$\begin{array}{lc}\text { CPDPRG/2 } & \text { waltz16 } \\ \text { PCPD2 } & 80.00 \text { usec }\end{array}$

$F 2$
$S I$

$\begin{array}{ll}S I & 32768 \\ S F & 100.6127727 \mathrm{MHz}\end{array}$

WDW $\because E M$

$\begin{array}{llll}S S B & 0 & \cdots & 1.00 \mathrm{H}\end{array}$

$\begin{array}{lll}L B & 0 & \\ G B & & \end{array}$
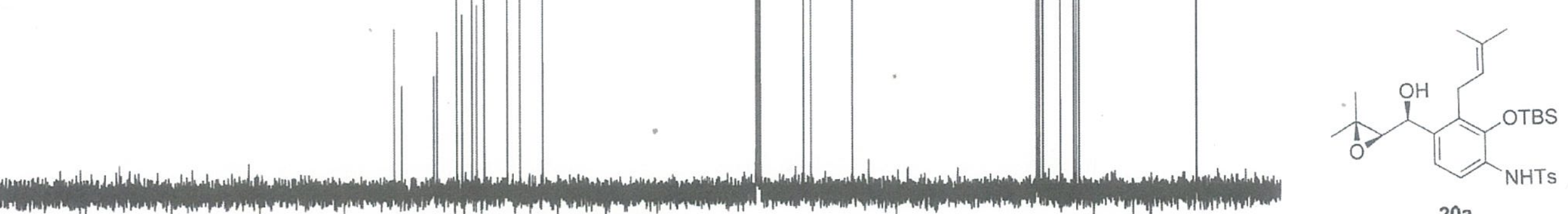

$20 a$

${ }^{13} \mathrm{C}-\mathrm{NMR}\left(100 \mathrm{MHz}, \mathrm{CDCl}_{3}\right)$

$\begin{array}{lllllllllllllllllllllll}210 & 200 & 190 & 180 & 170 & 160 & 150 & 140 & 130 & 120 & 110 & 100 & 90 & 80 & 70 & 60 & 50 & 40 & 30 & 20 & 10 & 0 & p p m\end{array}$ 


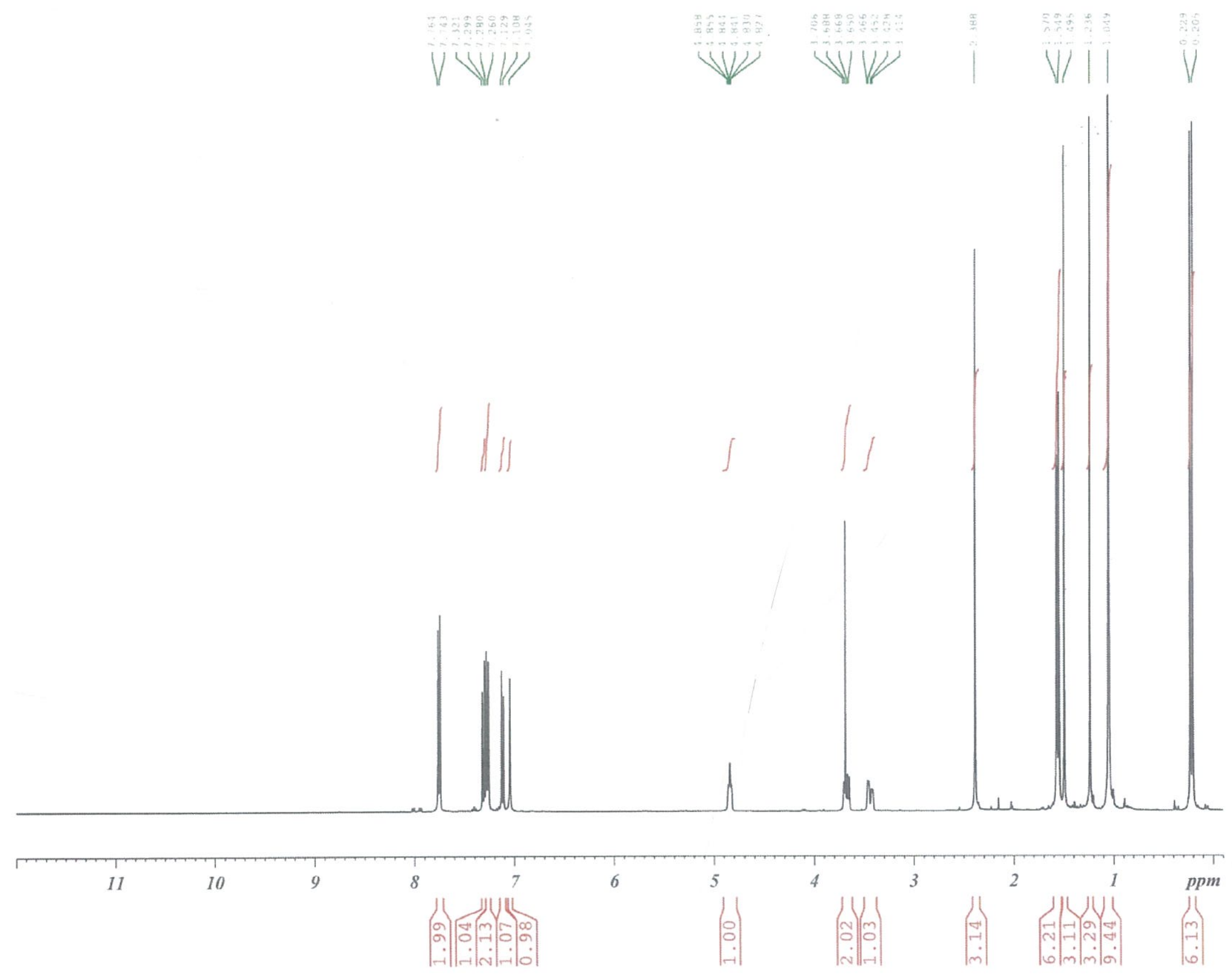

Current Data Parumeters
NAME
EXPNO ${ }_{\text {onB B BOOD }}$
PROCNO
PRO

F2-Acquisition Parameters
Date 20180615

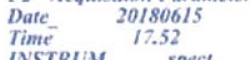

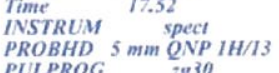

PULPROG ${ }_{65536}{ }^{2 H 30}$

SOLVENT ${ }_{\mathrm{N}} \mathrm{CDCl}$

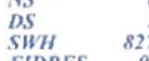

$\begin{array}{ll}\text { FWW } & 8278.146 \mathrm{~Hz} \\ \text { FIDRES } & 0.126314 \mathrm{~Hz} \\ \text { AO } & 0.9583745 \mathrm{sec}\end{array}$

$\begin{array}{ll}R \bar{R} & 57 \\ D W & 60.400 \text { usec }\end{array}$

$\begin{array}{cc}D E & 6.50 \text { usec } \\ T E & 297.2 \mathrm{~K} \\ \text { TE } & 1.00000000 \mathrm{sec}\end{array}$

TDO $\quad 1.00000000$ s.

$\overline{N U C l}=\underset{H H}{C H A N E L I}=$

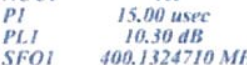

$F 2-$ Processing parameters
$S I$

$S F$
$W D W$$\quad \begin{array}{lll}32.1300107 \\ \text { EM }\end{array}$

$\begin{array}{lll}S S B & 0 & 0.30 \mathrm{H} \\ L B & 0\end{array}$

$\begin{array}{lll}L B & 0 & 0.30 \mathrm{H} \\ P C & & \end{array}$

7.00

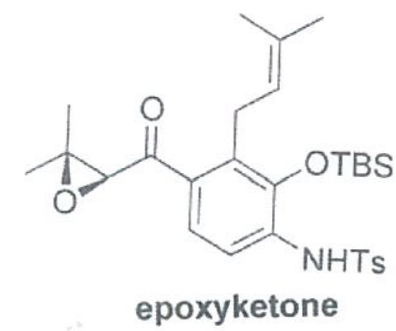

${ }^{1} \mathrm{H}-\mathrm{NMR}\left(400 \mathrm{MHz}, \mathrm{CDCl}_{3}\right)$ 


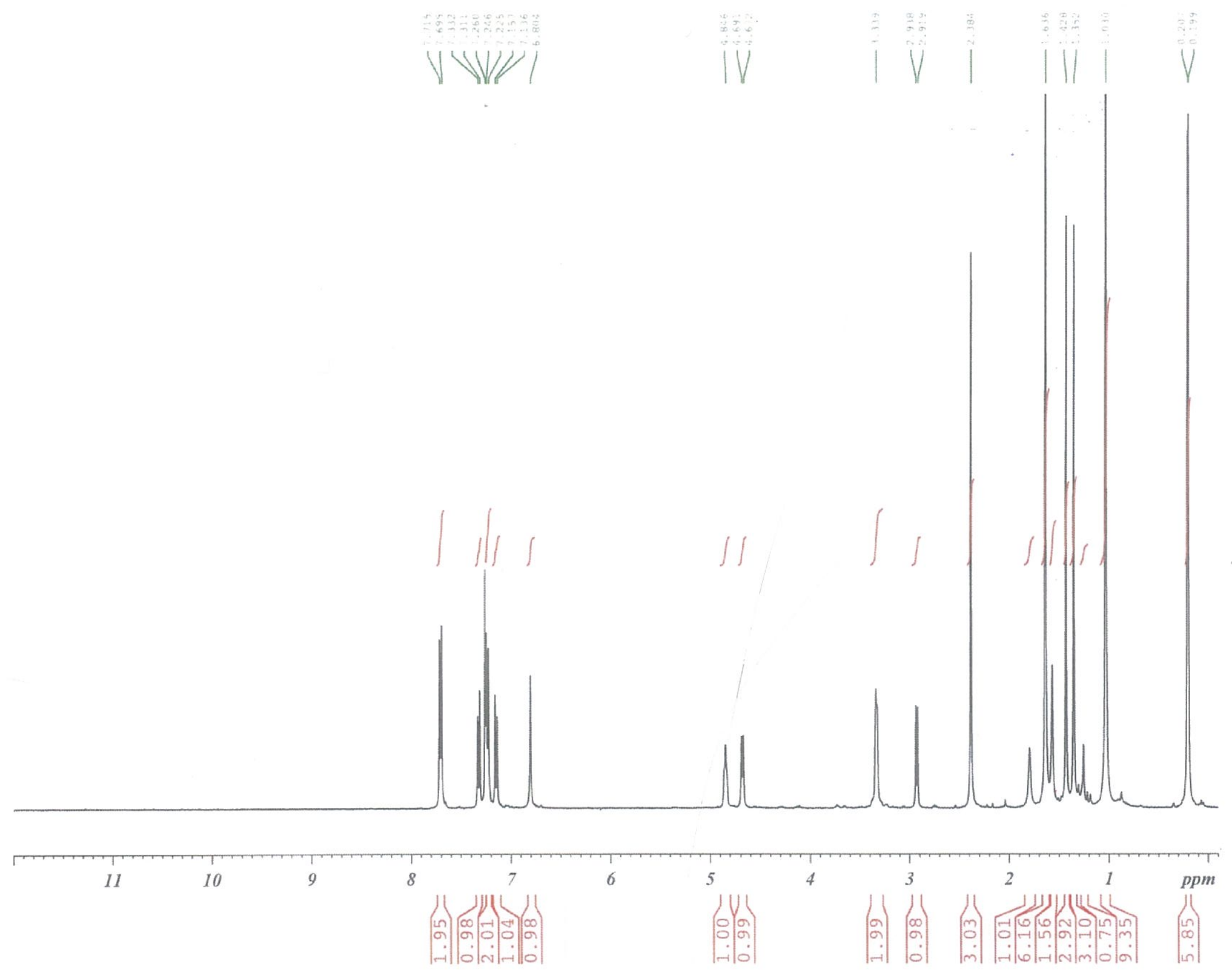

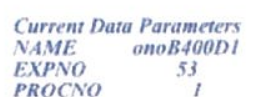

$F 2$ - Acquisition Parameters
Date
20180626

Time

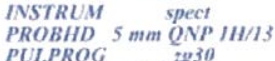

${ }_{T D}{ }^{2 L P R O G}{ }_{65536^{2330}}$

SOLVENT CDCB

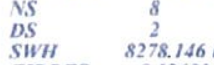

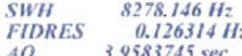

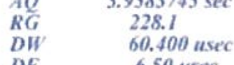

$\begin{array}{ccc}D E & 6.50 \text { usec } \\ T E & 297.2 \mathrm{~K} \\ D I & 1.00000000 \mathrm{sec}\end{array}$

$\overline{\text { NUCl }} \underset{I H}{C H A N I=}$

PI 15.00 useet

$\begin{array}{lll}\text { PLII } & 10.30 \mathrm{~dB} \\ \text { SFOI } & 00.1324710 \mathrm{MHz}\end{array}$

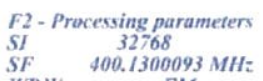

WDS $O B M$

$L B$
$G B$
$P C$

1.00

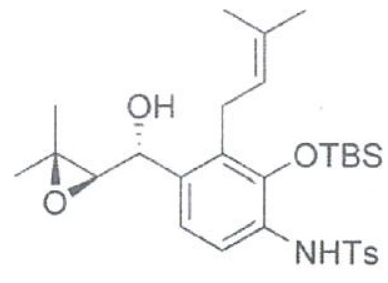

20b

${ }^{1} \mathrm{H}-\mathrm{NMR}\left(400 \mathrm{MHz}, \mathrm{CDCl}_{3}\right)$ 


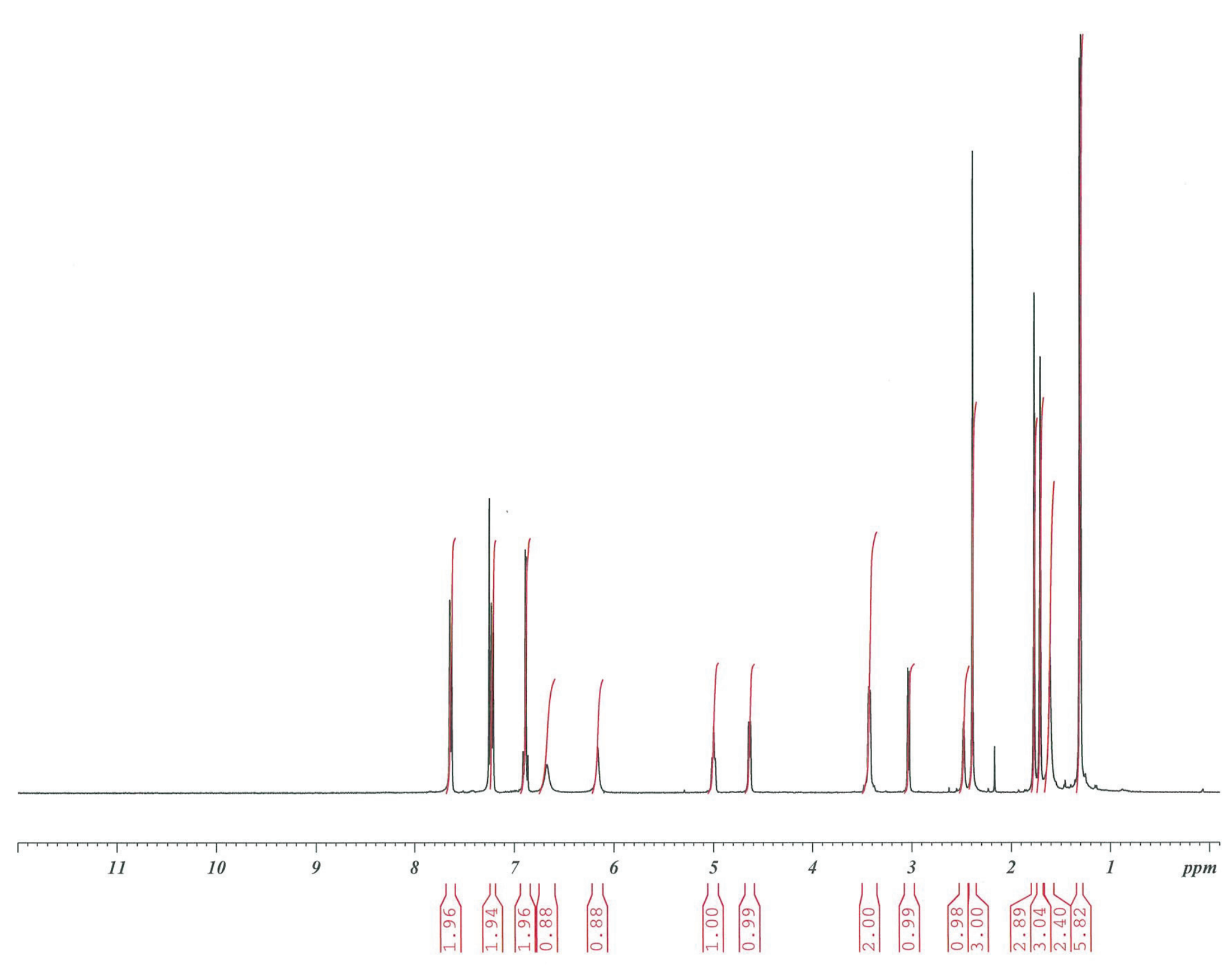

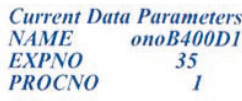

F2-Acquisition Parameter:
Date 2018008

$\begin{array}{lr}\text { Date } & 20180608 \\ \text { Time } & 12.59 \\ \text { INSTRUM } & \end{array}$

INSTRUM
PROBHD
PULPROG
PUL
QNP

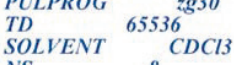

$N S$
$D S$

\begin{tabular}{ll}
$S W H$ & $8278.146 \mathrm{~Hz}$ \\
FIDRES & $0.126314 \mathrm{~Hz}$ \\
\hline &
\end{tabular}

$\begin{array}{ll}A Q & 3.9583745 \mathrm{sec} \\ R G & 3325\end{array}$

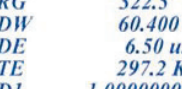

$\begin{array}{lc}T E & 297.2 \mathrm{~K} \\ D I & 1.0000000 \mathrm{~s} \\ T D 0 & 1\end{array}$

$\begin{array}{ccc} & \text { CHANNELL } \\ \text { PUI } & 1 \mathrm{H} \\ \mathrm{PI} & 15.00 \mathrm{us}\end{array}$

$\begin{array}{lc}\text { PI } & 15.00 \mathrm{usec} \\ \text { PLI } & 10.30 \mathrm{~dB} \\ \text { SFOI } & 400.1324710 \mathrm{MHz}\end{array}$

F2- Processing parameters

$\begin{array}{lc}S F & 00.1300093 \mathrm{MHz} \\ \text { WDW } & E M\end{array}$

$\begin{array}{lll}S S B & 0 & 0.30 \\ L B & & \end{array}$

$\begin{array}{lll}G B & 0 & 1.00\end{array}$

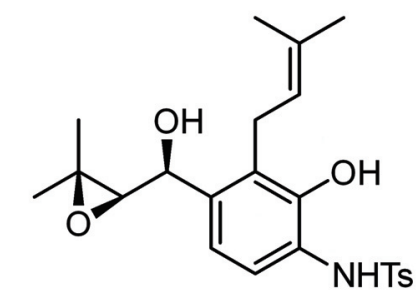

(+)-21b

${ }^{1} \mathrm{H}-\mathrm{NMR}\left(400 \mathrm{MHz}, \mathrm{CDCl}_{3}\right)$

$180608 \mathrm{Tf}$

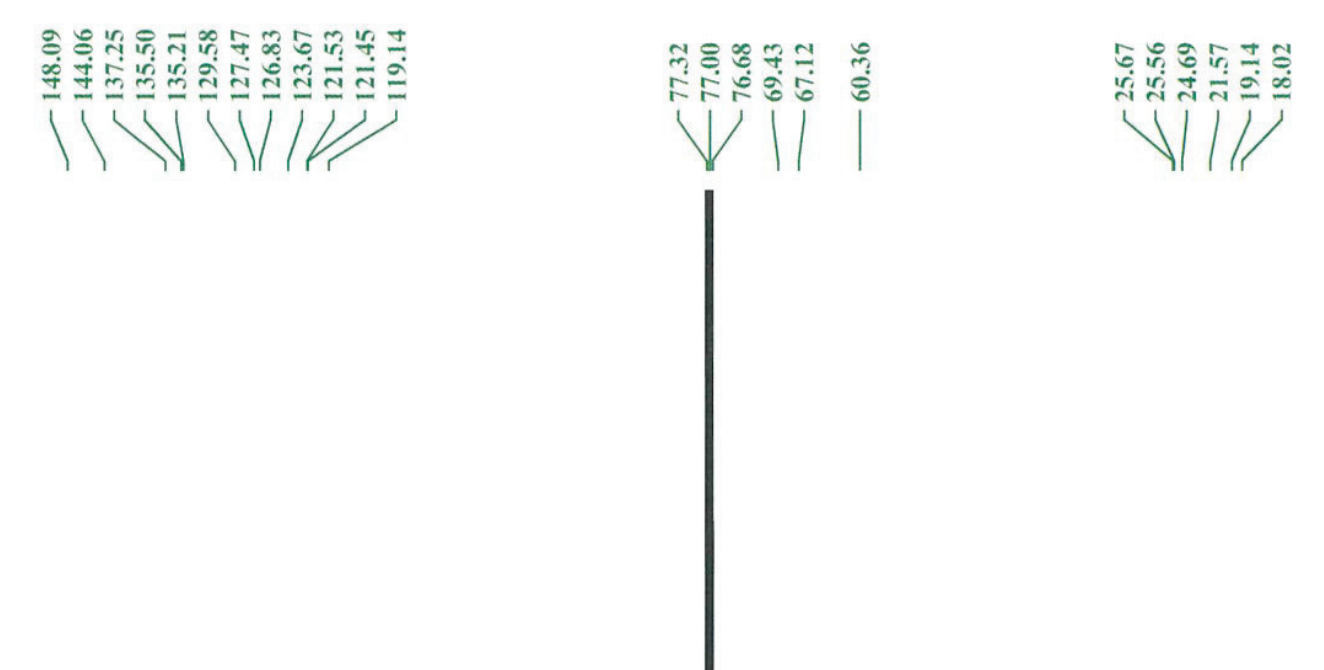

Current Data Parameters
NAME
EXPOBOA
OnPODI

F2 - Acquisition Parameter

$\begin{array}{cc}\text { Date } & 20180608 \\ \text { Time- } & 14.10\end{array}$

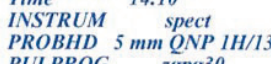
TDLPROG 655336 SOLVENT ${ }_{\text {NS }}{ }_{1024}^{C D}$ $\begin{array}{ll}D S & 2 \\ \text { SWH } & 23980.814 \mathrm{H}\end{array}$ $\begin{array}{ll}\text { SWH } & 23980.814 \mathrm{~Hz} \\ \text { FIDRES } & 0.365918 \mathrm{H} \\ \text { 10 } & 3664256 \mathrm{sec}\end{array}$ $\begin{array}{cc}A Q & 1.3664256 \mathrm{sec} \\ R G & 2298.8 \\ D W & 20.850 \mathrm{sec}\end{array}$

$D W$
$D E$
$T E$
$D E$

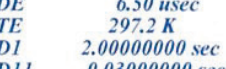

$D 11$
$T D O$

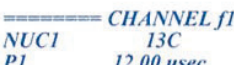

$\begin{array}{lll}12.00 \text { usec } & \\ P & 1\end{array}$

$\begin{array}{lll}P L I & 7.50 \mathrm{~dB} \\ \text { SFO } & 100.6228298 \mathrm{MHz}\end{array}$

$={ }_{C P D P R G / 2}$ CHANNEL $f 2$

NUC2 ${ }_{\text {NCPD2 }}$ IH

$\begin{array}{ll}P L 2 & 10.30 \mathrm{~dB} \\ P L 12 & 25.00 \mathrm{~dB}\end{array}$

$\begin{array}{lc}\text { PL12 } & 25.00 \mathrm{~dB} \\ \text { PL13 } & 25.00 \mathrm{~dB} \\ \text { SFO2 } & 400.1316005 \mathrm{MH}\end{array}$

FI - Processing parameters

$\begin{array}{ll}S I & 33768 \\ S F & 100.6127708 \mathrm{MHz}\end{array}$

$S S B \quad 0 \quad \mathrm{MM}$

$\begin{array}{lll}L B & 0 & 1.00 \mathrm{H} \\ G \mathrm{~B} & 0 & 1.40\end{array}$

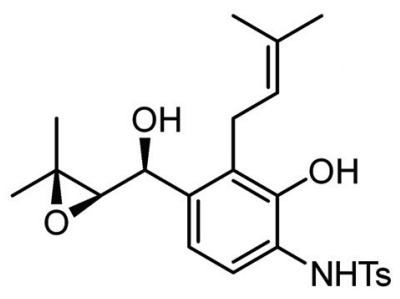

$+)-21 b$

${ }^{13} \mathrm{C}-\mathrm{NMR}(100 \mathrm{MHz}, \mathrm{CDCl} 3)$

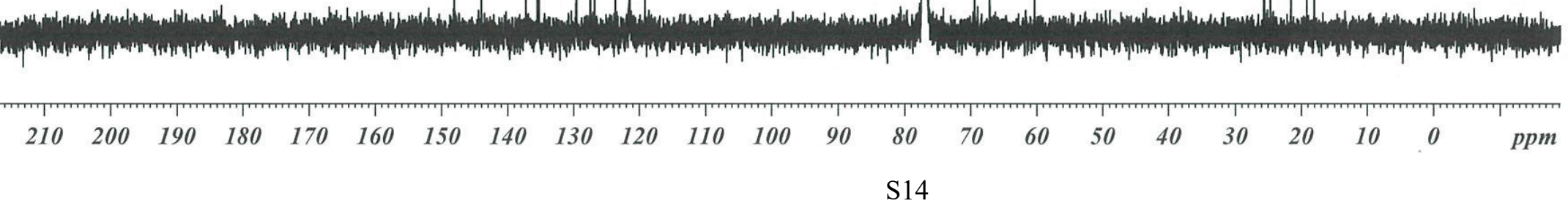




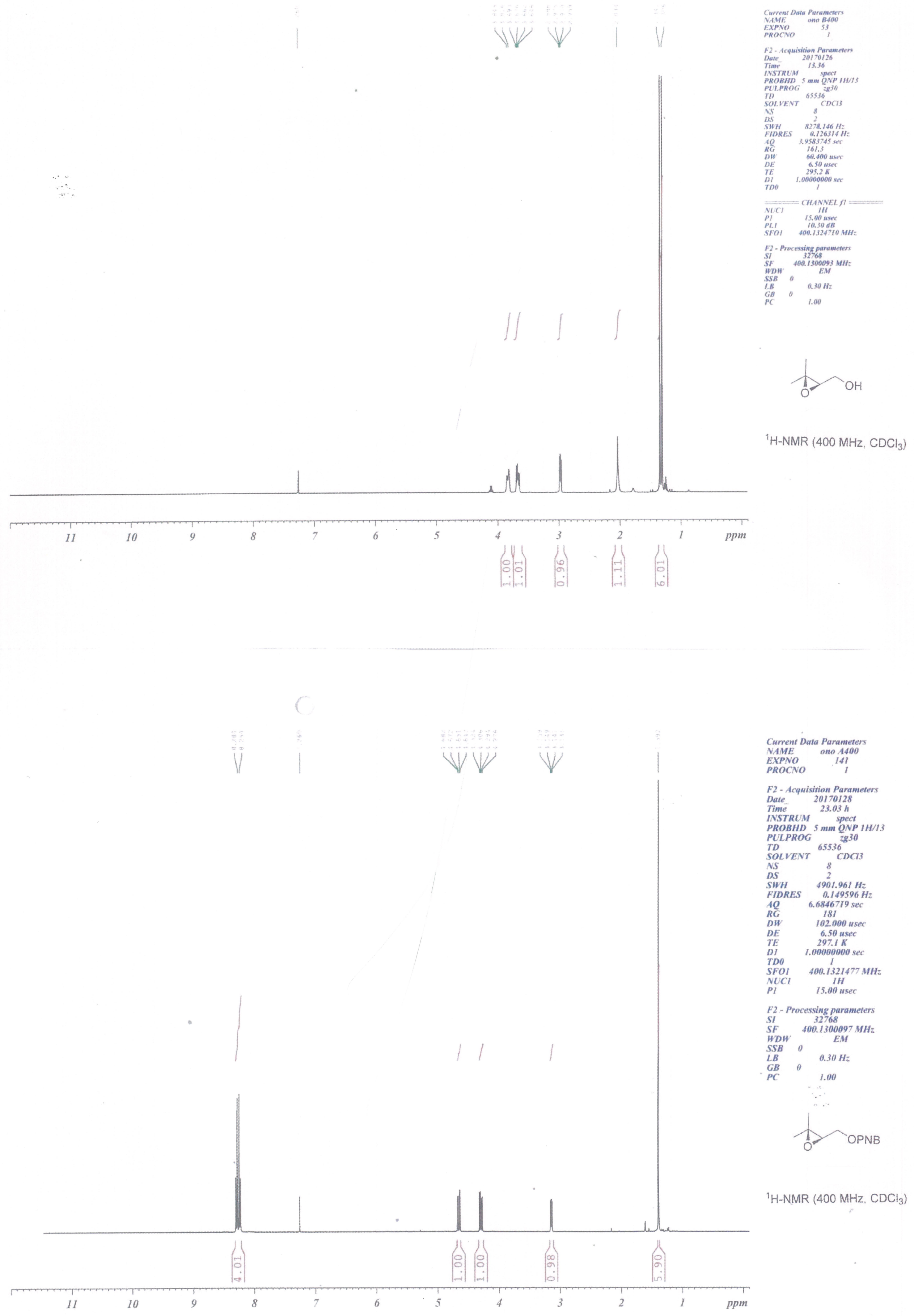




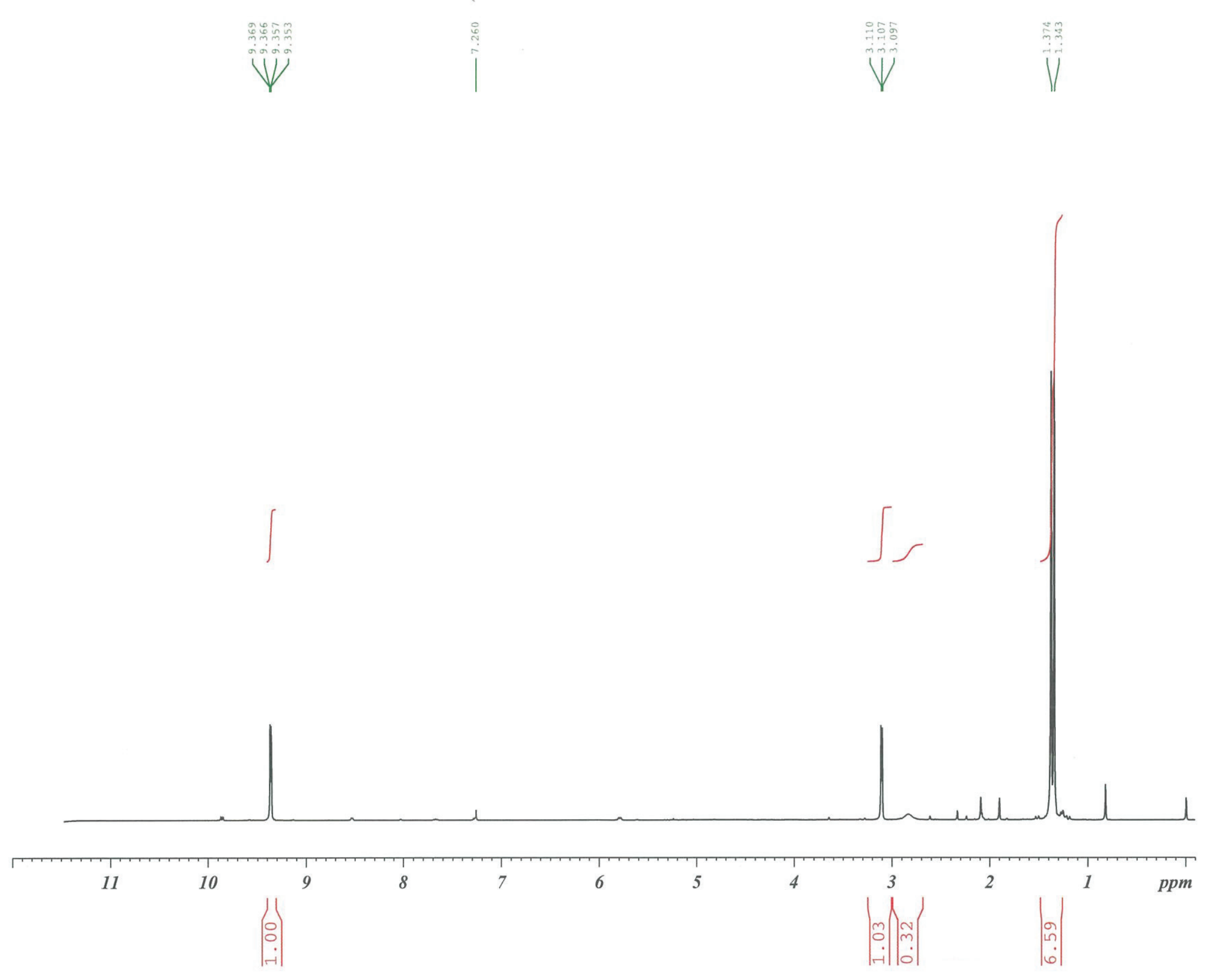

Current Data Parameters

NAME OnOA400DI

CNO 1

F2 - Acquisition Parameters

$\begin{array}{ll}20180512 \\ \text { Time } & 16.55 \mathrm{~h}\end{array}$

STRUM spect

ROBHD $5 \mathrm{~mm}$ QNP 1H/13

Pg30

TD ${ }^{65536}$

$\begin{array}{ll}N S & 8 \\ D S & 2\end{array}$

SWH $\quad 4901.961 \mathrm{~Hz}$

FIDRES $\quad 0.149596 \mathrm{~Hz}$

$\begin{array}{ll}A Q & 6.6846719 \mathrm{sec} \\ R G & 35.9\end{array}$

$D W \quad 102.000$ use

TE $296.9 \mathrm{~K}$

D1 $\quad 1.00000000 \mathrm{sec}$

$\begin{array}{lc}\text { TDO } & 1 \\ \text { SFO1 } & 400.1321477 \mathrm{MH}\end{array}$

NUC1 400.1321477

$\begin{array}{ll}\text { PI } & 15.00 \text { use }\end{array}$

F2 - Processing parameters

SF $\quad 400.1300083 \mathrm{MHz}^{2}$

WDW $\quad E M$

$\begin{array}{lll}S S B & 0 & 0.30 \mathrm{~Hz}\end{array}$

1.00

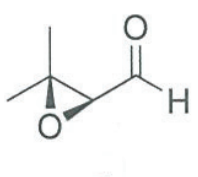

${ }^{1} \mathrm{H}-\mathrm{NMR}\left(400 \mathrm{MHz}, \mathrm{CDCl}_{3}\right)$

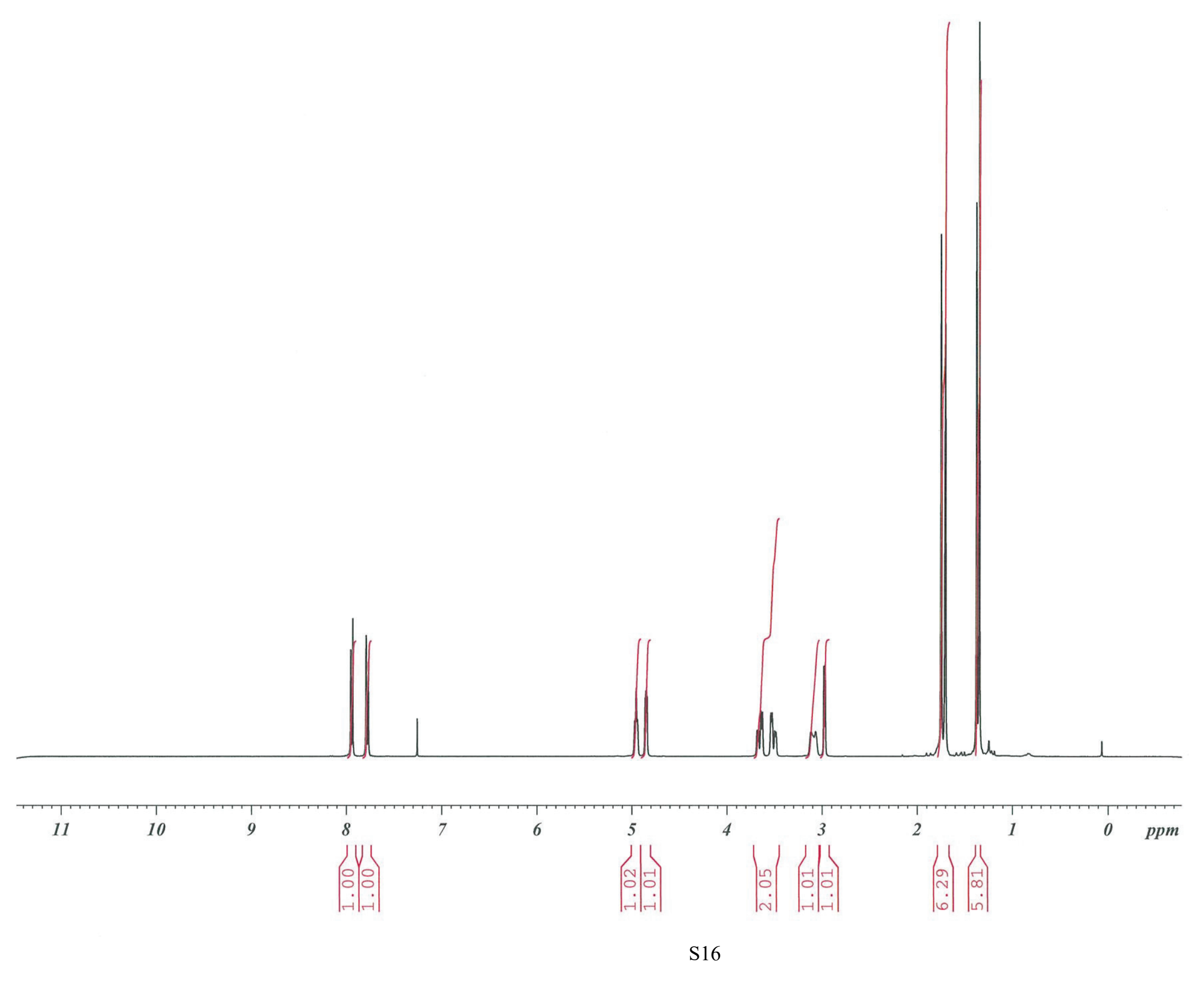

Current Data Parameters
NAME onoA400DI

EXPNO

PROCNO 1

- Acquisition Parameters

Date_ 20180705

TST

spect

${ }^{2 g 3}$

SOLVENT CDCl

$N S$

$\begin{array}{ll}D S & 2 \\ \text { SWH } & 4901.961 \mathrm{~Hz}\end{array}$

RG $\quad 70.6871$

DW $\quad 102.000$ usec

DE $\quad 6.50$ usec

$\begin{array}{lc}T E & 296.5 \mathrm{~K} \\ D I & 1.00000000 \mathrm{sec}\end{array}$

$1.00000000 \mathrm{sec}$
DDO

SFO1 $\quad 400.1321477 \mathrm{MH}$

$\begin{array}{lll}N O C l & 1 H \\ P 1 & 15.00\end{array}$

F2 - Processing parameter:

32768 or

WDW $400.1300092 \mathrm{MHz}$

SSB 0

$L B \quad 0.30 \mathrm{~Hz}$

$P C$

1.00

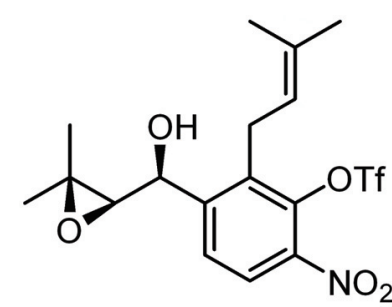

(士)-23

${ }^{1} \mathrm{H}-\mathrm{NMR}\left(400 \mathrm{MHz}, \mathrm{CDCl}_{3}\right)$ 
$\begin{array}{lc}\text { NS } & 128 \\ \text { SWH } & 2 \\ \text { SWI } & 23980.814\end{array}$

$\begin{array}{lr}\text { SWH } & 23980.814 \mathrm{~Hz} \\ \text { FIDRES } & 0.365918 \mathrm{~Hz}\end{array}$

$\begin{array}{lc}A Q & 1.3664256 \mathrm{sec} \\ R G & 2580.3 \\ D W & 20850 \mathrm{sec}\end{array}$

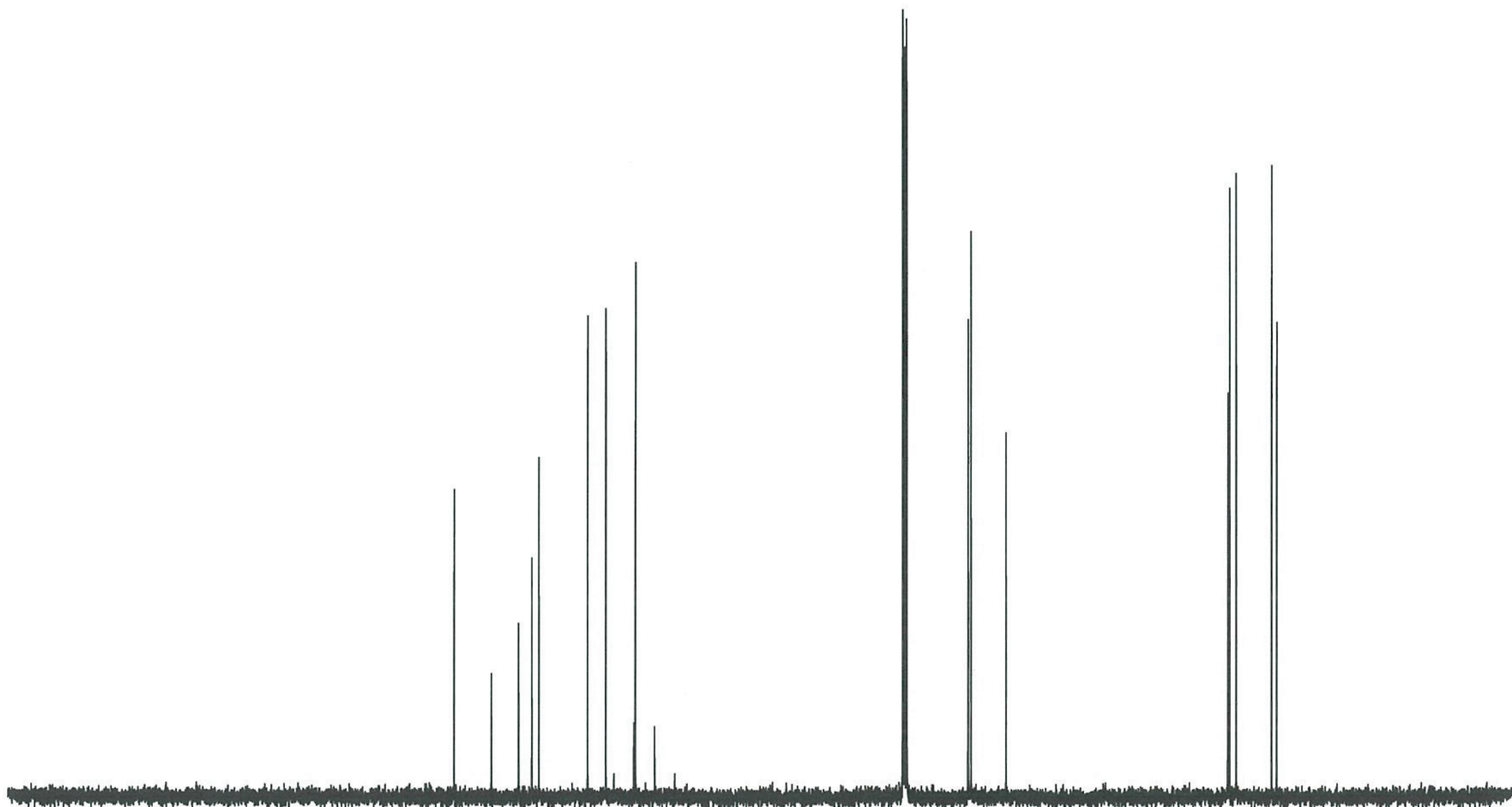

$\begin{array}{lll}\mathrm{NUCl} & \text { CHANNEL } \\ \mathrm{PI} & 13 \mathrm{C}\end{array}$

12.00 usec
$P_{L 1}$

SFOI $100.6228298 \mathrm{MH}$ $\underset{\text { NUC2 }}{\text { CPDPRG/2 }}$ CHANNEL $\begin{array}{ll}P L 2 & 10.30 d B \\ P L 12 & 25.00 d B\end{array}$ $\begin{array}{lc}\text { PLI2 } & 25.00 \mathrm{~dB} \\ \text { PLI3 } & 25.00 \mathrm{~dB} \\ \text { PLO2 } & 400.1316005 \mathrm{M}\end{array}$ F2 - Processing parameters
$S I$
$S F \quad 102768$
$S F=0127725 \mathrm{MHz}$

$\begin{array}{lll}L B & 0 & 1.00 \mathrm{H}\end{array}$

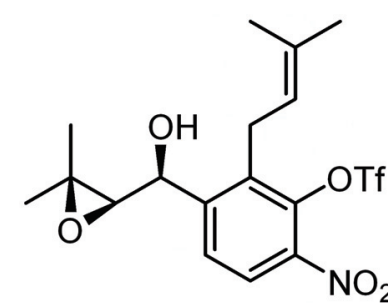

$( \pm)-23$

${ }^{13} \mathrm{C}-\mathrm{NMR}(100 \mathrm{MHz}, \mathrm{CDCl} 3)$

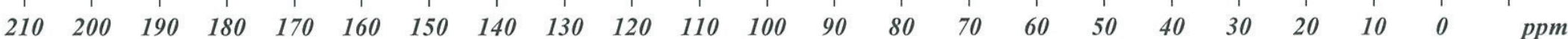

190405 no2 tf for data

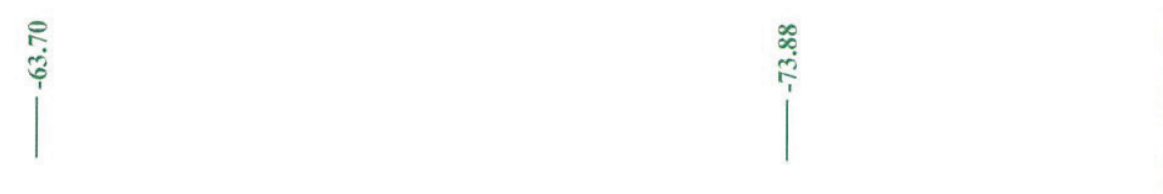

Current Data Parameters

EXPNO $\quad$ OHOA400D

PROCNO

F2 - Acquisition Parameters

$\begin{array}{ll}\text { Tate- } & 20190405 \\ & 16.28 \mathrm{~h}\end{array}$

NSTRUM spect

PROBHD $5 \mathrm{~mm}$ QNP $1 \mathrm{H} / 13$

PULPROG zgfhigqn

SOLVENT

DS

\begin{tabular}{ll} 
SWH & \multicolumn{2}{c}{$10964.912 \mathrm{~Hz}$}
\end{tabular}

E $\quad 0.669245 \mathrm{~Hz}$

$\begin{array}{ll}A Q & 1.4942209 \mathrm{sec} \\ R G & 5160.6\end{array}$

$D W \quad 45.600 \mathrm{usec}$

$\begin{array}{ll}D E & 6.50 \text { usec } \\ \text { TE } & 297.7 \mathrm{~K}\end{array}$

D1 $\quad 5.00000000 \mathrm{sec}$

$\begin{array}{lll}d 11 & 0.03000000 \mathrm{sec}\end{array}$

TDO 1

SFOI $376.4746466 \mathrm{MH}$

NUC1 $\quad 19 \mathrm{~F}$

$\begin{array}{lc}P 1 & 20.00 \mathrm{usec} \\ \text { SFO2 } & 400.1320007 \mathrm{MHz}\end{array}$

NUC2 $\quad 1 \mathrm{H}$

CPDPRG/2 waltz16

F2 - Processing parameters

$\begin{array}{lc}\text { SI } & 16384 \\ S F & 376.4987327 \mathrm{MHz}\end{array}$

$\begin{array}{lc}\text { SF } & 376.4987327 \mathrm{MH} \\ \text { WDW } & E M\end{array}$

$\begin{array}{lll}L B & 0 & 2.00 \mathrm{~Hz}\end{array}$

$\begin{array}{lll}G B & 0 & \\ P C & & 1.00\end{array}$

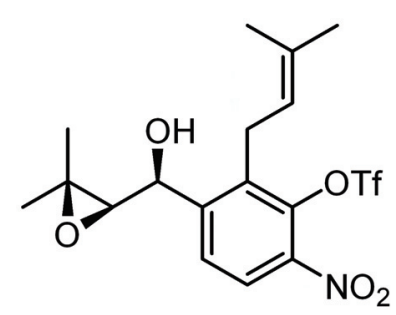

$( \pm)-23$

${ }^{19}$ F-NMR (376 MHz, CDCl3)

\begin{tabular}{|c|c|c|c|c|c|c|c|c|c|c|c|c|c|}
\hline \multirow{2}{*}{$\begin{array}{c}1 \\
-50\end{array}$} & 1 & 1 & 56 & 1 & 1 & 1 & 1 & 1 & 1 & 1 & 1 & 1 & 1 \\
\hline & -52 & -54 & -56 & -58 & -60 & -62 & -64 & -66 & -68 & -70 & -72 & -74 & -76 \\
\hline
\end{tabular}



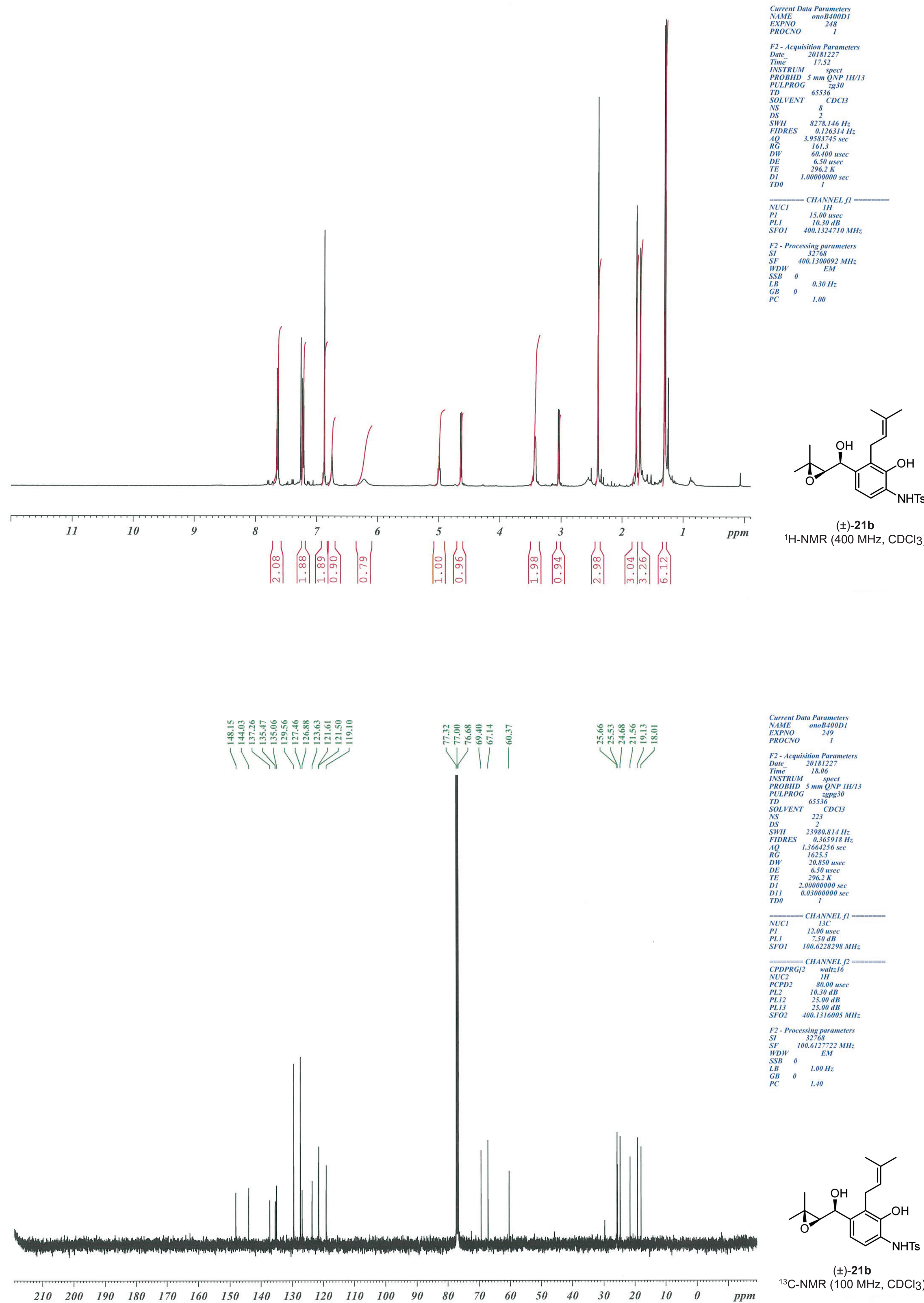

( \pm )-21b

${ }^{13} \mathrm{C}-\mathrm{NMR}(100 \mathrm{MHz}, \mathrm{CDCl} 3)$ 


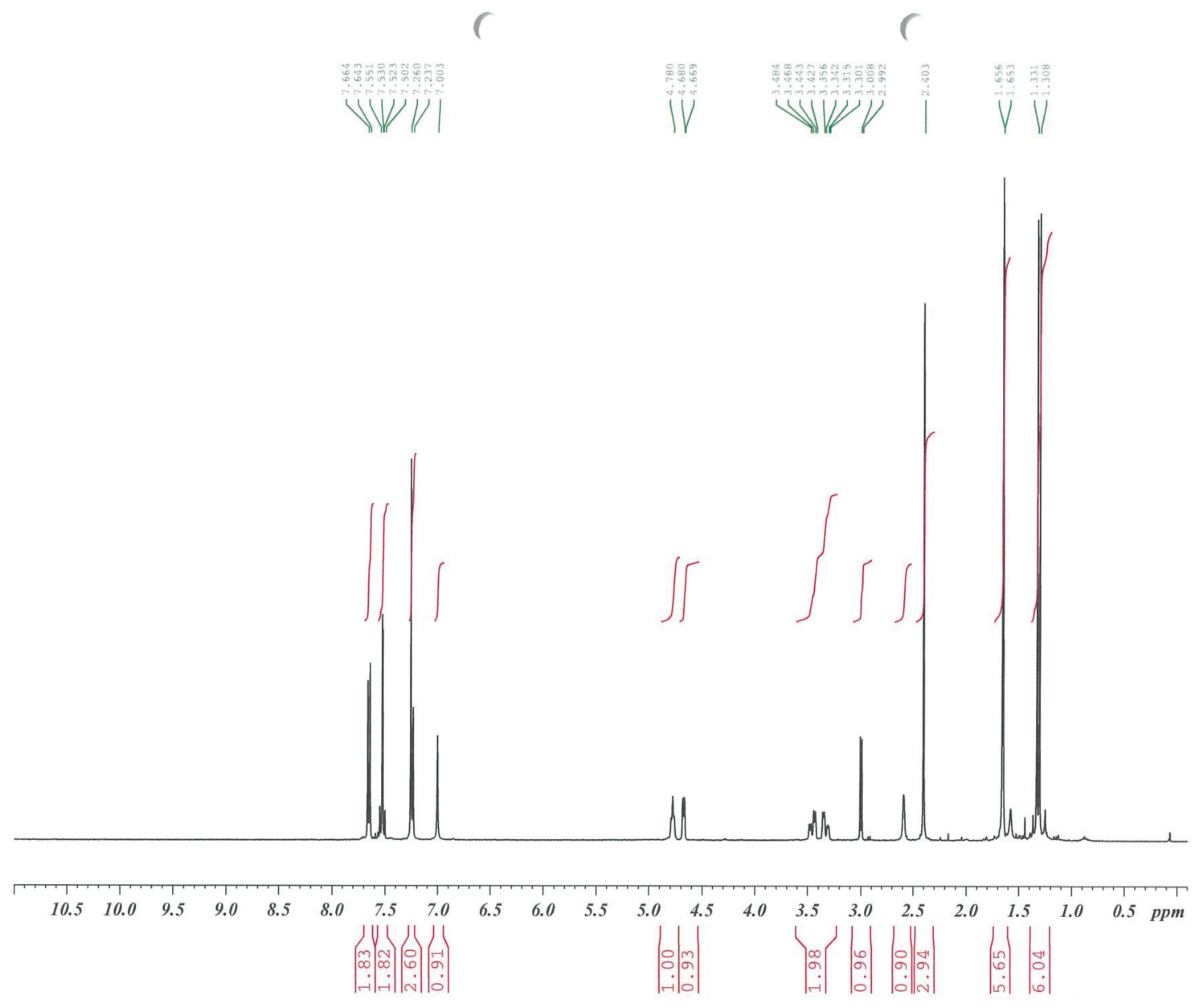

Current Data Parameters
NAME onoA400D2

PXPNO

F2 - Acquisition Parameters

Date_ 20190411

Time $18.48 \mathrm{~h}$

PROBHD $5 \mathrm{~mm}$ QNP IH/13

PULPROG

TD ${ }^{65536}{ }^{20 C l 3}$

${ }_{N S}^{\text {SOLVENT }}{ }_{8} \mathrm{CDCl3}$

NS

$\begin{array}{lc}\text { DS } & 2 \\ \text { SWH } & 4901.961 \mathrm{~Hz}\end{array}$

$0.149596 \mathrm{H}$
$\mathrm{AO}$

$R G \quad 362$

DW $\quad 102.000 \mathrm{usec}$

$\begin{array}{rr}D E & 6.50 \mathrm{us} \\ T E & 296.8 \mathrm{~K}\end{array}$

$\begin{array}{lc}\text { TDO } & 1 \\ \text { SFO1 } & 400.1321477 \mathrm{MHz}\end{array}$

$\begin{array}{lc}\mathrm{NUCl} & 1 \mathrm{H} \\ \mathrm{Pl} & 15.00 \mathrm{usec}\end{array}$

F2 - Processing parameters

$\begin{array}{lc}S I & 32768 \\ S F & 400.1300113 \mathrm{MHz}\end{array}$

EMW

$\begin{array}{lll}S S B & 0 & 0.30 \mathrm{~Hz}\end{array}$

${ }_{P C}^{G B}$

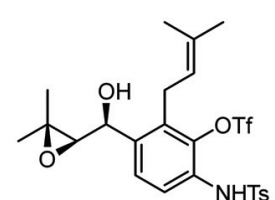

(+)-6

${ }^{1} \mathrm{H}-\mathrm{NMR}\left(400 \mathrm{MHz}, \mathrm{CDCl}_{3}\right)$

Synthesized from 21b in $76 \%$ yield

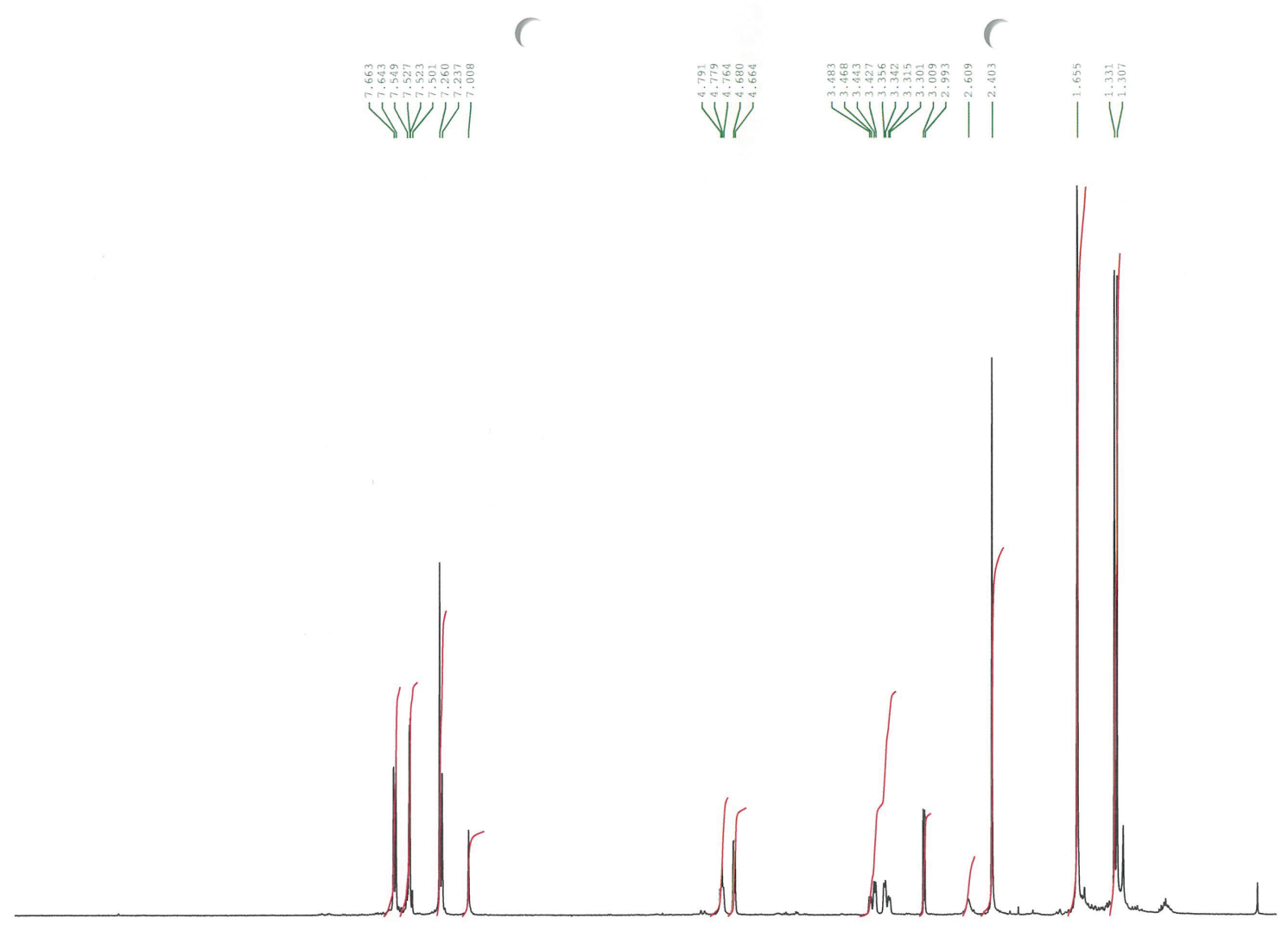

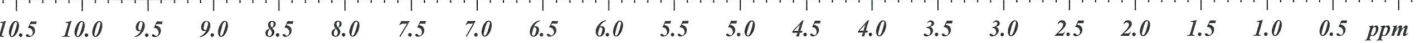

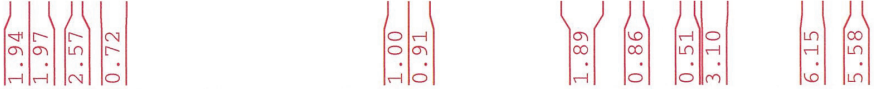
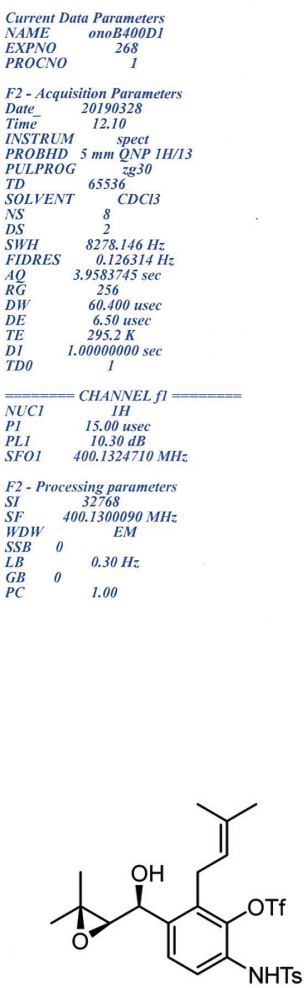

(+)-6

1 $\mathrm{H}-\mathrm{NMR}(400 \mathrm{MHz}, \mathrm{CDCl} 3)$

Synthesized from 20a in $65 \%$ yield ( 2 steps) 


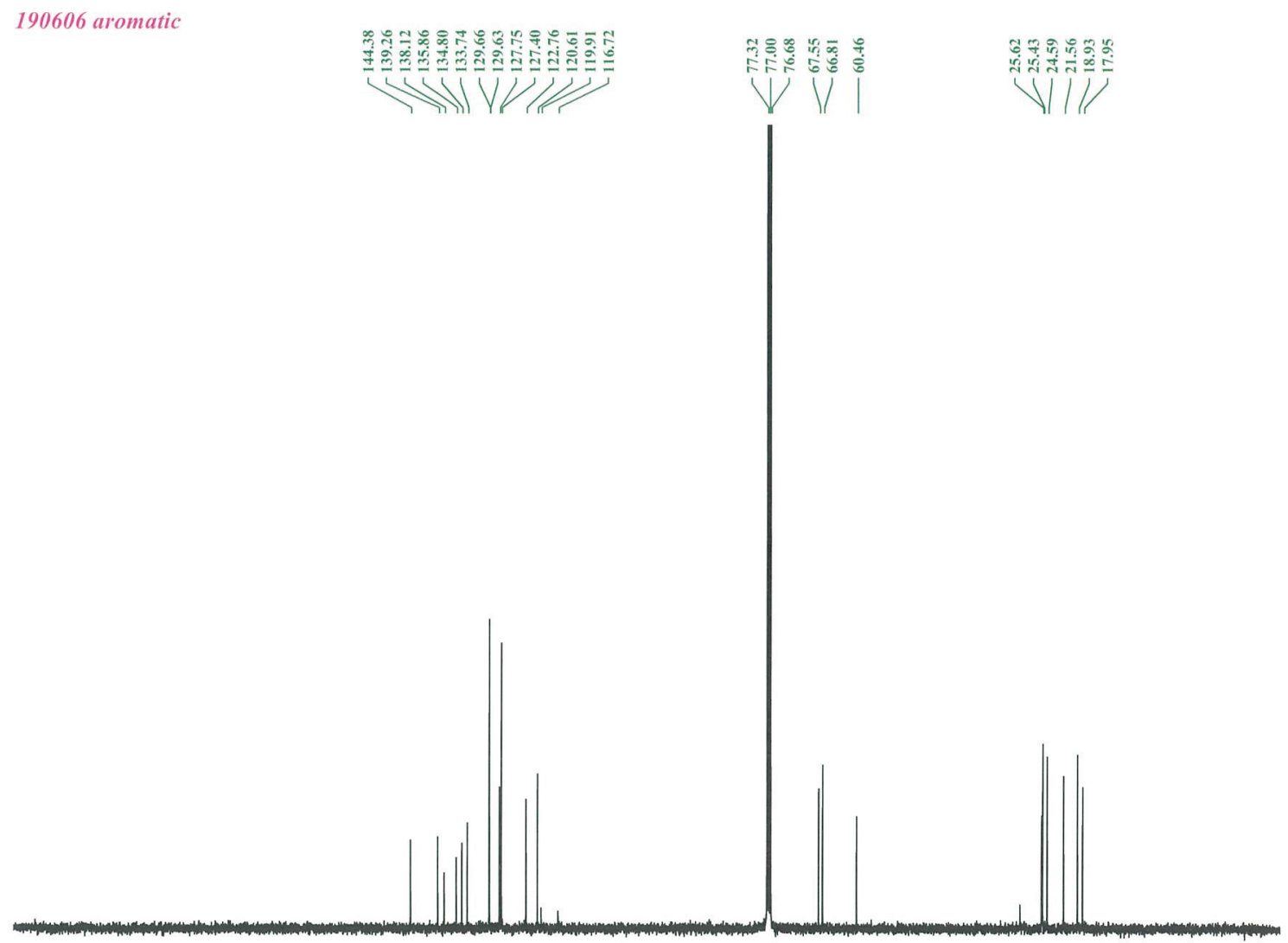

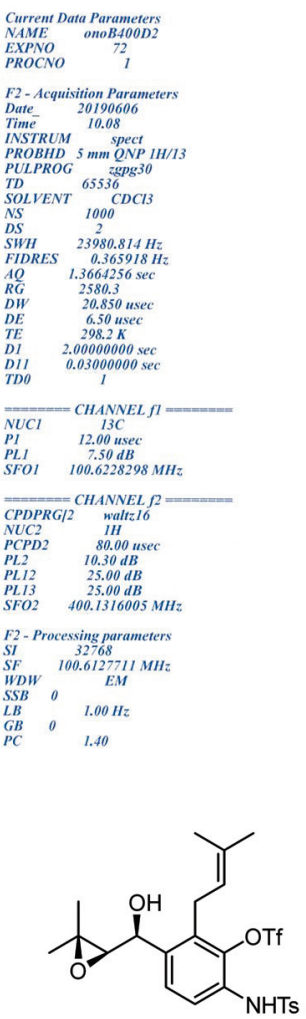

(+)-6

${ }^{13} \mathrm{C}-\mathrm{NMR}\left(100 \mathrm{MHz}, \mathrm{CDCl}_{3}\right)$
20190404 Tf for data

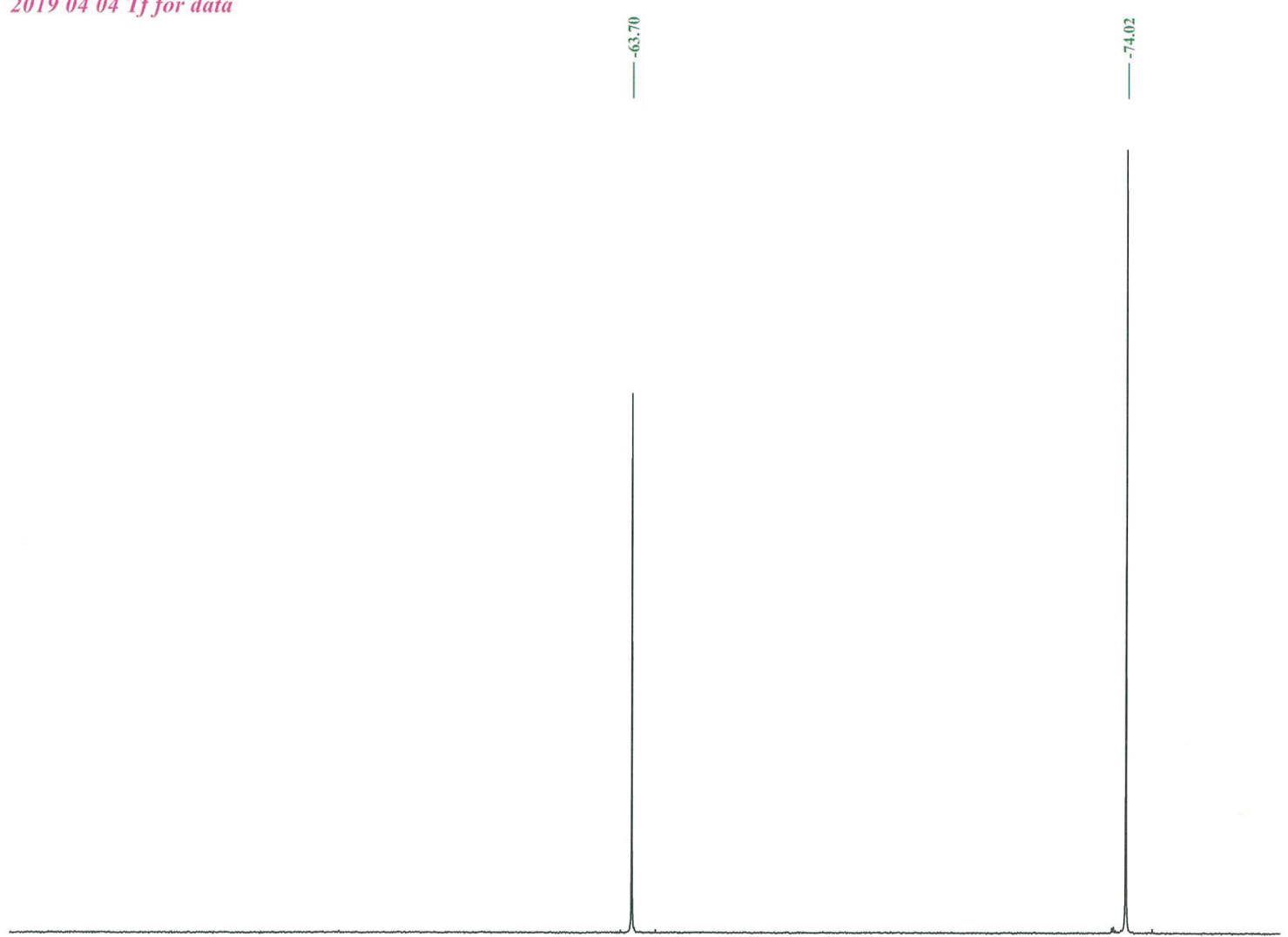

Current Data Parameters
NAME onoA400D2 $\begin{array}{lc}\text { NAME } & \text { OnOA400D } \\ \text { EXPNO } & 13 \\ \text { PROCNO } & 1\end{array}$

F2 - Acquisition Parameters $\begin{array}{cc}\text { Date } & 20190404 \\ \text { Time- } & 16.54 \mathrm{~h}\end{array}$ Time
INSTRUM $16.54 \mathrm{~h}$ PROBHD $5 \mathrm{~mm}$ QNP $1 \mathrm{H} / \mathrm{H} / 3$ PULPROG zgfhigqn TDOLVENT $\stackrel{32768}{\mathrm{CDCl}}$ NS 8 NS $\begin{array}{ll}\text { DS } & 2 \\ \text { SWH } & 9980.040 \mathrm{~Hz}\end{array}$ FIDRES $\quad 0.609133 \mathrm{~Hz}$ $\begin{array}{ll}A Q & 1.6416768 \mathrm{sec}\end{array}$ $\begin{array}{ll}R G & 13004 \\ D W & 50.100 \text { usec }\end{array}$ DE $\quad 6.50$ usec DI $\quad 5.00000000 \mathrm{sec}$ $3000000 \mathrm{sec}$ TDO $0.0000000 \mathrm{sec}$ SFO1 $376.4746466 \mathrm{MHz}$ $\begin{array}{lc}\text { NUCI } & 19 \mathrm{~F} \\ \text { P1 } & 20.00 \text { usec }\end{array}$ $\begin{array}{lc}\text { SFO2 } & 20.001 \mathrm{usec} \\ \text { ST0.1320007 } \mathrm{MHz}\end{array}$ NUC2 $1 \mathrm{H}$ $\begin{array}{lc}\text { CPDPRG/2 } & \text { waltz } 16 \\ \text { PCPD2 } & 80.00 \text { usec }\end{array}$

F2 - Processing parameters $\begin{array}{ll}S I & 16384 \\ S F & 376.4987362 \mathrm{MHz}\end{array}$ $\begin{array}{ll}L B & 2.00 \mathrm{~Hz}\end{array}$ $G B \quad 0 \quad 1.00$

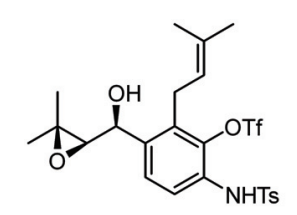
(+)-6

19F-NMR (376 MHz, CDCl3)

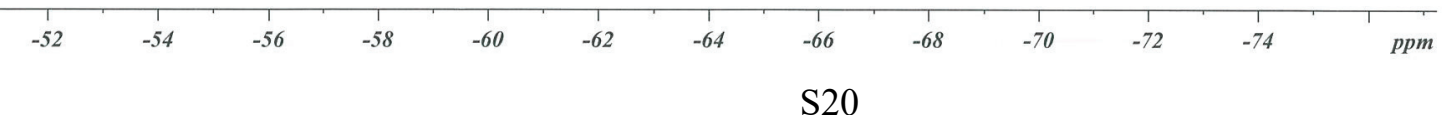




\section{References}

1. Martin, V. S.; Woodard, S. S.; Katsuki, T.; Yamada, Y.; Ikeda, M.; Sharpless, K. B. Kinetic resolution of racemic allylic alcohols by enantioselective epoxidation. A route to substances of absolute enantiomeric purity? J. Am. Chem. Soc. 1981, 103, 6237-6240.

2. Corey, E. J.; Bakshi, R. K.; Shibata, S.; Chen, C. P.; Singh, V. K. A stable and easily prepared catalyst for the enantioselective reduction of ketones. Applications to multistep syntheses. J. Am. Chem. Soc. 1987, 109, 7925-7926.

3. (a) Enders, D.; Zhu, J.; Raabe, G. Asymmetric Epoxidation of Enones With Oxygen in the Presence of Diethylzinc and (R,R)-N-Methylpseudoephedrine. Angew. Chem. Int. Ed. 1996, 35, 1725-1728.

(b) Enders, D.; Zhu, J.; Kramps, L. Zinc-Mediated Asymmetric Epoxidation of $\alpha$-Enones. Liebigs Ann. 1997, 1101-1113. (c) This epoxidation was successfully employed in two total syntheses of citrinadin A, a complex indole alkaloid possessing an epoxy prenyl group. See: Bian, Z.; Marvin, C. C.; Martin, S. F. Enantioselective Total Synthesis of (-)-Citrinadin A and Revision of Its Stereochemical Structure. J. Am. Chem. Soc. 2013, 135, 10886-10889. Kong, K,; Enquist, Jr., J. A.; McCallum, M. E.; Smith, G. M.; Matsumaru, T.; Menhaji-Klotz, E.; Wood, J. L. An Enantioselective Total Synthesis and Stereochemical Revision of (+)-Citrinadin B. J. Am. Chem. Soc. 2013, 135, 10890-10893.

4. Salvi, L.; Kim, J. G.; Walsh, P. J. Practical Catalytic Asymmetric Synthesis of Diaryl-, Aryl Heteroaryl-, and Diheteroarylmethanols. J. Am. Chem. Soc. 2009, 131, 12483-12493. 